UW Biostatistics Working Paper Series

$11-30-2009$

\title{
Pragmatic Estimation of a Spatio-Temporal Air Quality Model With Irregular Monitoring Data
}

Paul D. Sampson

University of Washington - Seattle Campus, pds@stat.washington.edu

Adam A. Szpiro

University of Washington, aszpiro@u.washington.edu

Lianne Sheppard

University of Washington, sheppard@u.washington.edu

Johan Lindström

Lund University, johanl@maths.lth.se

Joel D. Kaufman

University of Washington, joelk@u.washington.edu

\section{Suggested Citation}

Sampson, Paul D.; Szpiro, Adam A.; Sheppard, Lianne; Lindström, Johan; and Kaufman, Joel D., "Pragmatic Estimation of a SpatioTemporal Air Quality Model With Irregular Monitoring Data" (November 2009). UW Biostatistics Working Paper Series. Working Paper 353.

http://biostats.bepress.com/uwbiostat/paper353

This working paper is hosted by The Berkeley Electronic Press (bepress) and may not be commercially reproduced without the permission of the copyright holder.

Copyright (c) 2011 by the authors 
Paul D. Sampson ${ }^{1 *}$

Adam A. Szpiro ${ }^{2}$

Johan Lindström ${ }^{1,3}$

Joel D. Kaufman ${ }^{4}$

${ }^{4}$ Department of Environmental and Occupational Health Sciences, University of Washington,

\author{
${ }^{1}$ Department of Statistics, University of Washington, U.S.A.
}

${ }^{2}$ Department of Biostatistics, University of Washington, U.S.A.

${ }^{3}$ Centre for Mathematical Sciences, Lund University, Sweden

Correspondence to: Paul D. Sampson, Department of Statistics, University of Washington, Box 354322, Seattle,

WA 98195-4322, U.S.A. E-mail: pds@stat.washington.edu

Although the research described in this presentation has been funded in part by the United States

23 Environmental Protection Agency through grant R831697 and assistance agreement CR-83407101 to the University

24 of Washington, it has not been subjected to the Agency's required peer and policy review and therefore does not

25 necessarily reflect the views of the Agency and no official endorsement should be inferred. Additional support was

26 provided by an award to the University of Washington under the National Particle Component Toxicity (NPACT)

27 initiative of the Health Effects Institute (HEI). 


\section{Introduction}

Statistical analyses of the health effects of air pollution have increasingly used GIS-based

3 covariates for prediction of ambient air quality in "land-use" regression models. More recently

4 these regression models have accounted for spatial correlation structure in combining monitoring

5 data with land-use covariates. The current paper builds on these concepts to address spatio-

6 temporal prediction of ambient concentrations of particulate matter with aerodynamic diameter

7 less than $2.5 \mu \mathrm{m}\left(\mathrm{PM}_{2.5}\right)$ on the basis of a model representing spatially varying seasonal trends

8 and nonstatonary spatial correlation structures. Our hierarchical methodology provides a

9 pragmatic approach that fully exploits regulatory and other supplemental monitoring data which

10 jointly define a complex spatio-temporal monitoring design.

11 The specification of a modeling approach depends on a number of factors that vary with the

12 details and scientific purpose of the study in which the predicted exposures will be computed.

13 Among these are:

14 - The spatial resolution and scale of the monitoring data, including the number and spacing of

15 monitors and the spatial extent of the modeling domain. Different strategies may be

16 appropriate depending on whether one is addressing an urban area, "mesoscale" regions, or

17 larger scale regions such as the United States east of the Mississippi River.

18 - The temporal scales of monitoring data, with hourly, daily, 2-week, and monthly scales

19 appropriate in different situations.

20 - The characteristics of the monitoring devices, which may vary for data from multiple

21 monitoring networks.

22 - The characteristics of relevant spatio-temporal covariates, including their spatial and 23 temporal scales.

24 Our work has been developed for the Multi-Ethnic Study of Atherosclerosis and Air 25 Pollution (MESA Air). MESA Air is a cohort study funded by the U.S. Environmental Protection 26 Agency (EPA) that emphasizes accurate prediction of intra-urban variation in individual 
exposures to ambient air pollutants in order to accomplish its primary aim of assessing the

2 relationship between chronic exposure to air pollution and sub-clinical cardiovascular disease.

3 The MESA Air cohort includes more than 6000 male and female subjects in six major U.S.

4 metropolitan areas (Los Angeles, CA; New York, NY; Chicago, IL; Minneapolis-St. Paul, MN;

5 Winston-Salem, NC; and Baltimore, MD). The primary MESA Air hypotheses relate to chronic

6 exposure to $\mathrm{PM}_{2.5}$. We note that final exposure estimates in MESA Air will integrate predictions

7 of outdoor concentrations with additional subject-level data, including time-activity patterns,

8 home infiltration characteristics, address history, and employment address (Cohen et al., 2009).

9 Our aim here is to provide point spatial predictions of long-term average concentrations at

10 the residences of MESA Air subjects. A primary source of monitoring data is the EPA's

11 regulatory Air Quality System (AQS) repository (EPA 2010). The AQS network includes a

12 number of fixed site monitors in each region, each of which measures ambient air pollution

13 levels on a regular basis, either daily, every third day or every sixth day in the case of $\mathrm{PM}_{2.5}$.

14 Although there are some missing data, most AQS sites provide nearly complete $\mathrm{PM}_{2.5}$

15 concentration time series over several years at their spatial locations. The analysis here uses data

16 for the time period 2000-2006.

17 The MESA Air study conducted supplemental air quality monitoring campaigns beginning in

182005 to provide additional concentration data in order to better model and predict intra-urban

19 variation in air quality not well-represented by the AQS regulatory monitoring network. The

20 objective of the MESA Air monitoring is to more completely sample a spatial design space that

21 emphasizes traffic-related pollution at small spatial scales (100s of meters) and to capture data at

22 actual subject home locations. For logistical reasons, the supplementary monitoring data are

23 sampled as two-week averages based on an unbalanced design that results in significant amounts

24 of missing data at many measurement locations (Cohen et al. 2009). In each region, the

25 monitoring through 2006 includes: (a) from two to five fixed site monitors providing up to 1.5

26 years of 2-week observations, and (b) rotating sets of 2-week observations at (typically) 4 subject

27 homes, with monitors moved every two weeks to cover a total of about 50 subject homes, each 
observed twice. MESA Air monitoring at fixed and home sites continued through July, 2009,

2 providing a larger for future modeling. The composite of the AQS and MESA Air monitoring

3 data provide a rich but highly irregular spatio-temporal monitoring database for analysis.

Statistical approaches to the modeling of spatio-temporal air quality monitoring data for ambient exposure estimation typically derive from models that decompose observations into spatio-temporal trend and spatio-temporal residuals, or variation around the trend. Even after specification of the scientific questions and the spatio-temporal scales of interest, different statisticians will hold different perspectives on how-much spatial and temporal structure is to be represented as trend and how much as residual. For example, considering just a single time

10 series of air quality data one may explicitly model a trend using iterated moving averages as in 11 the KZ filter (Rao and Zurbenko, 1994; Wise and Comrie, 2005), various state-space modeling 12 approaches (e.g., Taylor et al. 2006), spline smoothing, or simple parametric models such as 13 polynomial or trigonometric series. Alternatively, one can incorporate much of the variation in

14 the residuals by using a sufficiently rich autocorrelation model. Somewhat similar choices arise 15 in modeling spatial trends and residuals, with different perspectives provided by splines that 16 model the spatially correlated component semi-parametrically as part of the mean model and 17 geostatistical approaches that account for spatial structure not predicted by covariates using a 18 variogram model for the autocorrelation. However, there are mathematical frameworks that 19 allow one to show equivalence between certain spline and kriging predictions (e.g., Kent and 20 Mardia, 1994; Furrer and Nychka, 2007).

21 A number of characteristics of the variation in air-quality data across the urban scales of 22 interest in our current work motivate the modeling strategy and the approach to trend and 23 residual that we present. We find that all air quality measurements demonstrate systematic time 24 trends and seasonality, but these time trends vary in space, even over relatively small 25 metropolitan area spatial scales. The details of the trend and seasonality vary somewhat from 26 year to year, so that they are not modeled well by simple periodic functions like sinusoids or 27 other trigonometric functions. Finally, characteristics of these systematic time trends, including 
1 the long-term mean and amplitude of seasonal variations, covary with spatial (or "land use")

2 covariates. Even though the primary interest in MESA Air is predicting spatial variation of long-

3 term average concentrations to estimate exposures, the complex spatio-temporal monitoring

4 design necessitates a statistical modeling approach that accounts for spatio-temporal interactions

5 in the data. Otherwise we cannot use the spatially rich but temporally sparse data at MESA Air

6 homes to help in estimating the long-term averages.

7 For an overview of general techniques for modeling correlated spatio-temporal data, see

8 Banerjee et al. (2004). See also the closely related strategy of Le and Zidek (2006). Smith et al.

9 (2003) use an expectation-maximization (EM) algorithm to allow for arbitrary missing data

10 patterns, but their model does not accommodate the complex spatio-temporal interactions

11 addressed here. A recent paper by Fanshawe et al. (2008) demonstrates how carefully chosen

12 covariates may eliminate the need to accommodate spatio-temporal correlation in the residuals,

13 but the model in that paper assumes a uniform time trend across a relatively small spatial area.

14 Paciorek et al. (2009) and Sahu et al. (2006) model particulate matter using techniques that allow

15 for more complex spatio-temporal interactions, however their estimation and prediction

16 procedures (based on a different approach to representing spatio-temporal trend and residual) are

17 applicable only with a nearly complete (balanced) space-time monitoring data matrix.

18 The pragmatic modeling and prediction procedure described here includes sufficiently

19 complex spatio-temporal interactions to account for variation in seasonal patterns at different

20 locations, and it accommodates essentially arbitrary patterns of missing data with respect to an

21 ideally complete space by time matrix of observations. We use an extensive database of GIS-

22 based covariates in a spatio-temporal generalization of universal kriging in order to predict

23 spatial variation in seasonal trends and in two-week ambient concentration levels. We compute

24 predictions of concentrations at all MESA Air subject residences, and then compute estimates of

25 long-term average concentrations as empirical averages of the predicted time series at these

26 locations. 
In Szpiro et al. (2009) we describe a likelihood-based version of the hierarchical model

2 presented below and study its statistical properties by applying it to oxides of nitrogen $\left(\mathrm{NO}_{\mathrm{x}}\right)$ in a

3 simulation scenario based on a subset of the MESA Air geographic covariates, subject locations,

4 and monitoring data. Lindström et al. (2010) extend this modeling to incorporate predictions

5 from a source dispersion model as a spatio-temporal covariate, also for analyses of $\mathrm{NO}_{\mathrm{x}}$. The

6 present paper focuses on a multi-step pragmatic approach to estimation and utilizes a more

7 complete set of covariates and monitoring data to predict $\mathrm{PM}_{2.5}$ concentrations at the homes of

8 all MESA Air subjects. We use the Los Angeles study area as an example to illustrate the data

9 structure and modeling approach. In order to make predictions at all MESA Air subject homes,

10 we fit the model separately in four geographic regions covering the six MESA Air study areas:

11 Southern California, a Midwest region spanning Minneapolis-St Paul and Chicago, a Northeast

12 region spanning Baltimore and New York City, and North Carolina.

13 The outline of this paper is as follows. In Section 2 we describe the monitoring data as well

14 as the geographic covariates that are available to inform predictions. Section 3 describes our

15 hierarchical model, and Section 4 provides a detailed account of the pragmatic multi-step

16 estimation and prediction procedure. In Section 5 we apply our model to predict concentrations

17 at the home addresses of MESA Air subjects in all study areas. We illustrate the procedure using

18 data from the Los Angeles region, and we also show predictions for subjects in all six MESA Air

19 regions. Section 6 summarizes this work and discusses issues to be addressed in future research.

2. Data

\subsection{AQS and MESA Air Monitoring data}

We incorporate data from two types of AQS fixed site monitors, monitors recording $\mathrm{PM}_{2.5}$

23 concentrations daily and monitors recording concentrations every third day. We use all 247

24 available "non-source oriented" monitors in counties nearby MESA Air subject locations that

25 contain a minimum of at least 1 year of continuous data. We extended the spatial domain as

26 necessary to include a minimum of 20 monitors around each MESA Air study city. 
The MESA Air supplementary monitoring for $\mathrm{PM}_{2.5}$ in each of the six study areas collects two-week average concentrations under two different spatio-temporal sampling plans: one for "fixed sites" and one for "home outdoor" sites. All of the locations at which data had been collected in the California region as of Dec 31, 2006 are shown on the map in Figure 1.

There are a total of seven MESA Air fixed sites in the Los Angeles area, one of which is co-

7 located with an AQS monitor to allow for instrument calibration. These fixed sites began

8 measuring two-week average concentrations in November 2005. There were approximately 40

9 measurements per site and a total of 264 fixed site measurements during this timeframe. A total

10 of 45 home outdoor monitoring locations in Los Angeles are also included; these were sampled

11 during two-week periods starting in May 2006. The plan called for each home to be sampled two

12 times, in different seasons. (Not all homes were sampled twice before the end of 2006, the

13 closing date for the database for the analysis in this manuscript.) Figure 2 presents a schematic

14 illustration of the spatio-temporal sampling scheme combining the various AQS (EPA) and

15 MESA Air monitoring sites.

For this analysis all AQS data are summarized at the 2-week time scale of the MESA Air monitoring campaigns. One practical feature of this data structure is that 2-week mean pollutant concentrations have far simpler temporal structure than daily data, which demonstrate high

20 temporal autocorrelation, even after removing temporal trends. Figure 3 shows four example

21 time series on the 2-week time scale. We computed overlapping 2-week averages of $\mathrm{PM}_{2.5}$

22 concentrations from AQS monitoring sites because the MESA Air monitoring periods in the

23 Riverside area to the east were offset one week from the monitoring periods for central and

24 coastal Los Angeles. These 2-week averages are centered on the Wednesday midpoints of the

25 MESA Air 2-week sampling periods. We required at least 4 valid daily AQS observations for 26 computation of a 2-week mean (actually a mean over 15 days). For this preliminary analysis we 27 do not account for differences between monitor types or temporal sampling density (i.e. daily vs. 
1 every $3^{\text {rd }}$ day). Monitoring sites sampling only every $6^{\text {th }}$ day did not provide enough data to

2 estimate valid 2-week averages.

--- Figure 3. ---

The four $\mathrm{PM}_{2.5}$ series in Figure 3 present log transformations of two-week averages. The three AQS monitoring sites are located: in the Riverside area to the east (060658001), in the north central area of the Los Angeles concentration of MESA Air subjects (060372005), and to the northwest near the coast in Ventura County (061113001). We note similar, but slightly varying temporal trends as depicted by the smooth curves (explained in section 4) and variation in the long-term mean concentration which is highest in Riverside and lowest along the coast in Ventura County.

The final short time series in Figure 3 presents one of the MESA AIR fixed sites in the coastal area of Los Angeles County. The black time trend drawn on this plot is largely determined by an average of the trends from the AQS sites nearest this MESA AIR fixed site. The extrapolation of the time trend prior to the beginning of monitoring in 2006 has similar features to the trend curve for the central Los Angeles site, but is quite different from Ventura trend curve. Estimation of a long-term mean at MESA Air monitoring sites requires this spatially interpolated trend.

\subsection{GIS-based geographical covariates}

Our strategy for predicting concentrations at locations and times without measurements includes the use of regression models with geographic covariates. This is often termed "land use" regression (LUR) (Moore et al. 2007; Ross et al. 2007; Hoek et al. 2008). Our application of LUR is embedded in a hierarchical spatio-temporal model that incorporates flexible correlation structures. We consider a variety of geographic covariates, including: (i) indirect measures of traffic influences provided by distances to major roads (major roads identified by census feature class codes A1-A3), together with lengths of such roads in seven circular buffers from 50 to 750 meters around sites of interest, (ii) average population density (number of people per square $\mathrm{km}$ 
1 in the block group where the monitor or participant is located), and (iii) percentages of land in

2 circular buffers described by various land use categories such as commercial property, cropland,

3 industrial property, and residential property. These are all derived using the ArcGIS (ESRI,

4 Redlands, CA) software package. The population density is calculated from publicly available

5 U.S. Census Bureau data, and the roadway variables are derived from the proprietary TeleAtlas

6 Dynamap 2000 roadway network. In total we considered approximately 200 possible covariates

7 accounting for the road and land use variables measured in seven nested circular buffers.

8 Covariates were screened prior to analysis and those with essentially no variability in a given

9 study region (e.g. percent of forest in Los Angeles) were omitted. The number of covariates

10 remaining for analysis ranged from 41 for Southern California to 66 for the Northeast region

11 (including geographic coordinates derived from latitude and longitude).

\section{3. Components of the space-time hierarchical model}

13 Our statistical model is comprised of a spatio-temporal trend model and spatio-temporal

14 residuals. This decomposition of space-time concentrations (for log-transformed 2-week

15 averages) can be written

$$
Y_{s, t}=\mu(s, t)+\varepsilon(s, t)
$$

17 where $s$ indexes space and $t$ time, $\mu$ is the trend surface, and $\varepsilon$ is the residual surface. Our 18 approach accounts for spatial variability in temporal trends in order to make use of the complex

19 spatio-temporal monitoring data from the combined AQS and MESA Air monitoring campaigns

20 as well as a possibly nonstationary spatial covariance structure in the residuals . Our proposed

21 model includes sufficiently complex spatio-temporal interactions to account for both variations

22 in seasonal patterns at different locations and changes over time in the configuration of sites

23 available for spatial predictions. It accommodates the fact that air quality measurements

24 demonstrate systematic time trends and seasonality, that these time trends vary in space, even

25 over relatively small metropolitan area spatial scales, and that the details of the trend and 
seasonality vary somewhat from year to year (and, hence, are not modeled well by simple

2 sinusoids).

We write the temporal trend at location $s$ as a linear combination of $m$ smooth, orthogonal,

4 temporal basis functions $f_{j}(t)$ :

$$
\mu(s, t)=\beta_{0 s}+\sum_{j=1}^{m} \beta_{j s} f_{j}(t)
$$

We compute the $f_{j}(t)$ from data and refer to them as smoothed empirical orthogonal functions

7 (SEOFs) (Fuentes et al., 2006). These basis functions are defined to have temporal averages

8 equal to zero so that $\beta_{0 s}$ represents the long-term temporal mean. We will demonstrate that a

9 linear combination of a small number of these SEOFs is sufficient to characterize the variation in

10 temporal trends, resulting in residuals with negligible temporal correlation.

11 The coefficients of this model are the amplitudes of the temporal basis function patterns.

12 They are modeled to vary systematically in space according to regressions on $q$ spatial covariates 13 assembled into an $N \times q$ design matrix :

$$
\boldsymbol{\beta}_{j}=\left(\begin{array}{c}
\beta_{j s_{1}} \\
\vdots \\
\beta_{j s_{N}}
\end{array}\right) \sim N\left(\mathbf{X} \alpha_{j}, \Sigma_{e}\left(\phi_{j}\right)\right)=\sum_{k=1}^{q} X_{k} \alpha_{j k}+\mathbf{e}_{j}
$$

15 where $s_{1}, \ldots, s_{N}$ denote the spatial locations of the $N$ monitoring sites, and $X_{k}$ is the $N \times 1$ vector 16 of values on the $k^{\text {th }}$ spatial covariate with regression coefficients $\alpha_{j k}$. The spatial covariance 17 model, $\Sigma_{e}\left(\phi_{j}\right)$, needs to be estimated for each of the $j=1, \ldots, m$ spatial regressions (also called 18 universal krigings). We fit standard exponential spatial correlation functions with nugget effects. 19 We assume the spatio-temporal residuals $\varepsilon(s, t)$ are temporally independent but spatially 20 correlated with a common covariance for all time periods, written as

$$
\boldsymbol{\varepsilon}_{t}=\left(\begin{array}{c}
\varepsilon_{s_{1}, t} \\
\vdots \\
\varepsilon_{s_{N}, t}
\end{array}\right) \sim N\left(0, \Sigma_{\varepsilon}\left(\phi_{\varepsilon}\right)\right)
$$

22 where the spatial covariance matrix $\Sigma_{\varepsilon}\left(\phi_{\varepsilon}\right)$, with spatial correlation parameters $\phi_{\varepsilon}$, is computed

23 using the Sampson-Guttorp spatial-deformation model for nonstationary spatial covariance

24 (Damian et al., 2003). The applicability of this model is based on the assumption that the 
1 meteorological events that can drive high temporal autocorrelation structure on a daily time scale

2 are largely averaged out on the 2-week time scale of the MESA Air supplemental monitoring.

3 Our aim is to separate the spatially varying temporal trends from the residuals, thus leaving the

4 residuals from the trend essentially uncorrelated in time.

\section{4. Computational steps in estimation of the space-time model}

6 The steps described below are: (1) the computation of Smoothed Empirical Orthogonal

7 Basis functions (SEOFs), (2) the fitting of smooth temporal trends at each of the monitoring sites

8 using these SEOFs, (3) analysis of spatial variation in the fitted smooth temporal trends by a

9 Partial Least Squares approach to land use regression, (4) modeling of the spatial covariance

10 structure of the residuals from the fitted spatio-temporal trends, and (5) cross-validated

11 assessment of spatio-temporal predictions of $\mathrm{PM}_{2.5}$ using a spatio-temporal universal kriging

12 procedure (sometimes called "kriging with external drift").

\subsection{Smoothed Empirical Orthogonal basis Functions}

14 The first step in fitting our hierarchical model to data is to derive the smoothed empirical 15 orthogonal basis functions (SEOFs), $f_{j}(t)$, that we use to fit spatially varying temporal trend.

16 Sampson introduced an approach to computing SEOFs in Fuentes et al. (2006). We elaborate

17 this approach here. Consider a spatio-temporal matrix $\mathbf{Y}$ of $T$ observations (rows) at $N$

18 locations (columns) in space and suppose that we want to approximate $\mathbf{Y}$ as a linear combination

19 of $m$ SEOFs. We write

$$
\mathbf{Y}=\mathbf{M}+\mathbf{E}
$$

21 where

$$
\mathbf{M}=\mathbf{F} \boldsymbol{\beta},
$$

23 F being a $T \times m$ matrix with columns representing the values of $m$ temporal basis functions,

24 and $\beta$ being an $m \times N$ matrix of coefficients.

$$
\mathbf{F}=\left[f_{0}(t) f_{1}(t) \cdots f_{m}(t)\right]
$$




$$
\boldsymbol{\beta}=\left(\begin{array}{cccc}
\beta_{01} & \beta_{02} & \cdots & \beta_{0 N} \\
\beta_{11} & \beta_{12} & \cdots & \beta_{1 N} \\
\vdots & \vdots & \cdots & \vdots \\
\beta_{m 1} & \beta_{m 2} & \cdots & \beta_{m N}
\end{array}\right)
$$

2 The most parsimonious set of basis functions for a least squares minimization of $\mathbf{E}$ is

3 obtained by taking $\mathbf{F}$ to be the matrix of the first $m$ left singular vectors of the singular value

4 decomposition (SVD):

$$
\mathbf{Y}=\mathbf{U} \mathbf{D} \mathbf{V}^{\prime}
$$

6 That is, using the superscript $(m)$ to denote sub-matrices with $m$ columns, write

$$
\mathbf{Y}=\mathbf{U}^{(m)}\left(\mathbf{D}^{(m)}\left(\mathbf{V}^{(m)}\right)^{\prime}\right)+\mathbf{E}
$$

8 This suggests that we take the matrix of empirical orthogonal functions, $\mathbf{F}$, to be the matrix

9 of left singular vectors $\mathbf{U}^{(m)}$. However we wish our temporal basis functions to be defined as

10 smooth functions of time, and the usual left singular vectors will not vary smoothly over the row

11 (time) index. In addition, in practice every data matrix $\mathbf{Y}$ that we consider will have some

12 missing data so that we cannot simply compute the usual singular value decomposition. We

13 therefore embed the following "EM-like" procedure (algorithm SVD.em) for computation of an

14 "SVD" of a matrix with missing data in a cross-validated smoothing loop to derive SEOFs

15 (algorithm SEOF).

16 Algorithm SVD.em:

17 1. Specify a dimension (rank), $m$, for the model.

18 2. Scale the observations at each monitoring site (columns of $\mathbf{Y}$ ) to norm (variance)

19 one; call this matrix $\tilde{\mathbf{Y}}$.

20 3. Compute an initial average temporal vector $u_{1}$ as the set of row averages of nonmissing values in $\tilde{\mathbf{Y}}$. Initialize missing observations in the data matrix $\tilde{\mathbf{Y}}$ using elements of a rank-one approximation provided by a regression (without intercept) of the data in each column (site) on $u_{1}$.

4. Compute the rank- $m$ SVD-approximation of the now complete data matrix. 
5. Impute the missing values in $\tilde{\mathbf{Y}}$ by the elements of the rank- $m$ SVD approximation just computed.

6. Return to step 4 and iterate to convergence.

We specify the scaling in step 2 so that all sites contribute equally to the characterizing variability in patterns of temporal trend regardless of the amplitude of trends at the sites. This algorithm, coded in the R system (R Development Core Team, 2009), converges adequately fast in all of the applications we have faced. The computation of EOFs in the presence of missing data has also been addressed in the oceanographic literature (Beckers and Rixen, 2003).

As a practical approach to smoothing the EOFs, we compute smoothing spline regressions on the time index using the smooth.spline function in the R language (with generalized crossvalidation specified by the argument $\mathrm{CV}=\mathrm{F}$ ). To choose the dimension of the SEOF model we use the BIC criterion computed for predictions of trend in the following cross-validation loop:

Algorithm SEOF:

In a cross-validation loop leaving out a random subset of sites in each iteration:

1. Compute the SVD using the SVD.em algorithm above

6

2. Smooth vectors consisting of every other 2 -week value of $m$ left-singular vectors using smooth. spline (with generalized cross-validation specified by argument $\mathrm{CV}=\mathrm{F})$. Evaluate the fitted spline on every weekly observation using the R function predict.smooth.spline.

3. Compute the trend prediction of every site in the left-out cross validation group by least squares regression on the smoothed trend components and evaluate a BIC criterion for fit.

The smoothing in step (b) uses every other value so that the smoothing spline would not be applied to time series with temporal correlations artificially inflated by the overlapping of 2week averages. The smoothed singular vectors are no longer exactly orthogonal, but exact 
1 orthogonality is not a concern. We choose as optimal the number of components $m$ with best (or

2 near-best) distribution of BIC values in the cross-validated fits.

3 Although the SVD.em algorithm can be run on matrices with arbitrary amounts of missing

4 data, MESA Air home sites have too little data, only one or two observations, to permit

5 meaningful multiple regressions on the smoothed temporal basis functions. Furthermore, as

6 MESA Air fixed site monitors began operation in 2005 or 2006, these sites also have few

7 observations compared to the AQS sites. For this reason the specification of the temporal trend

8 functions just described was computed using only the AQS monitoring sites.

\subsection{Trend fits at MESA Air monitoring sites}

Once the SEOFs are determined, the next step in our pragmatic procedure is to estimate

11 values for the corresponding trend coefficients $\beta_{0 s}, \beta_{1 s}, \ldots, \beta_{m s}$ at each spatial location with

12 monitoring data. Since there are nearly complete temporal data at each AQS monitor, we

13 estimate the coefficients at these locations by linear regression of the data on the corresponding

14 SEOFs.

15 As the data at MESA Air sites are more limited, we fit trends at these locations using

16 information provided by AQS sites in a spatial neighborhood. In principal we might use a model

17 like that of Banerjee and Johnson (2006,), which we would call a "spatial random effects" model.

18 However, the fitting of that model as currently implemented is computationally impractical for

19 the size of our spatio-temporal datasets. We choose instead to use local random effects modeling

20 strategy so that the fitted trend at a MESA AIR site is a simple empirical Bayes fit with

21 shrinkage of trend model coefficients to the average trend of those AQS sites in a local

22 neighborhood. We chose neighborhood sizes of 25 to $40 \mathrm{~km}$ in different regions in order to

23 assure that at least three neighboring AQS sites were used in this fitting at each of the MESA Air

24 sites. The random effects regression models were computed by REML using the Imer function

25 of the Ime 4 package for the R system (http://cran.r-project.org/web/packages/lme4/). 
The shape of the temporal trends determined by the coefficients $\hat{\beta}_{0 s}, \hat{\beta}_{1 s}, \ldots, \hat{\beta}_{m s}$ computed

2 this way is necessarily determined almost entirely by the neighboring AQS sites as the MESA

3 Air time series are too short to carry much weight. To assure that the MESA Air observations

4 contribute as much as possible to estimation of the long-term average coefficients $\beta_{0 s}$, rather

5 than use the REML estimate of overall trend we used the estimated coefficients $\hat{\beta}_{1 s}, \hat{\beta}_{2 s} \ldots, \hat{\beta}_{m s}$,

6 to detrend the MESA Air observations, computing

$$
Y_{s t}^{d}=Y_{s t}-\sum_{j=1}^{m} \hat{\beta}_{j s} f_{j}(t)
$$

and then take the average of the detrended observations as an estimate of the long-term average

$$
\hat{\beta}_{0 s}=\frac{1}{T} \sum_{t} Y_{s t}^{d} .
$$

Fitted trends derived from trend coefficients $\hat{\beta}_{0 s}, \hat{\beta}_{1 s}, \ldots, \hat{\beta}_{m s}$ computed by this procedure

11 appear appropriate by visual inspection. In principal, estimated trend coefficients should be

12 influenced by the spatial covariates according to the regression model of equation (2). That is

13 the case in our likelihood-based approach to the hierarchical model (Szpiro et al., 2009), but

14 covariates are not incorporated at this stage of our sequential fitting procedure. Fitted trend

15 coefficients are used as outcomes in the computation of spatial regression models as explained in

16 the following sub-section.

\section{$17 \quad 4.3$ Spatial regression by Partial Least Squares (PLS)}

18 Our hierarchical model proposes that the spatial variation in the parameters $\hat{\beta}_{j s}$ of the 19 temporal trend models can be modeled via regression on spatial covariates. The GIS-based 20 dataset of spatial covariates for $\mathrm{PM}_{2.5}$ concentrations provides substantial numbers of highly

21 correlated spatial covariates. For example, the composite lengths of A1 roads in buffers of, say,

22400 meters around specified locations are highly correlated with lengths of roads in 500 meter

23 buffers and negatively correlated with the distance to the nearest A1 road. Percentages of

24 property in various land use categories are similarly correlated across buffer sizes; for example,

25 percentages of land classified residential in a buffer are substantially negatively correlated with

26 percentages of land classified as commercial. Model specification with large sets of 
multicollinear predictors typically involves either (a) variable selection, (b) shrinkage or regularization, perhaps including variable selection as in a "lasso" approach (Tibshirani, 1996), or (c) regression on a smaller number of composite covariate scores. While our fundamental concern is the quality of predictions, we prefer not to choose a method that would select one particular buffer size for inclusion in a model ignoring neighboring buffer sizes, or one particular land use categorization at the expense of a correlated land use categorization. We choose, instead, to regress on a small number of composite covariate scores using the method of PLS regression to define the composite scores (see, for example, Garthwaite, 1994 or Abdi, 2010).

9 The description of the composite scores in terms of individual variable loadings can be useful as

10 it facilitates comparison of regression models across the four geographic modeling regions. PLS 11 regressions were computed using the pls package for the R system (http://cran.r-

12 project.org/web/packages/pls).

13 Almost all the numeric land use covariates considered here have skewed distributions and 14 are log-transformed for analysis. Most of the spatial covariates fall into one of three groups: (1) 15 shortest distances to roads and commercial properties, (2) lengths of roads of different classes 16 (A1, A2, A3) within buffers, and (3) percentage of property in difference land use categories in 17 buffers. We have chosen to log-transform all of the numerical scores except for the "angle" to $18 \mathrm{~A} 1 / \mathrm{A} 2 / \mathrm{A} 3 \mathrm{road}$ variables and the "residential" land use variables, which span the entire 0-100 19 percent range. Log-transformations were computed after selection of an empirically determined 20 constant to add in order to deal with zero scores. To be considered for analysis we require a 21 covariate to have more than 5 non-zero observations.

22 The code in the pls package for the R system computes conventional leave-one-out cross23 validatory assessments of predictions of these regression models. However, these cross24 validations assume a modeling framework with spatially independent errors. Since our 25 hierarchical model includes spatial correlation, we choose the dimension of the PLS regression 26 by cross-validation with a universal kriging prediction involving component scores defined by 27 the PLS algorithm and a model for the spatial covariance structure of the residuals from those 
1 regressions as indicated in equation (2). We computed spatial covariance matrices $\Sigma_{e}\left(\phi_{j}\right)$ in

2 terms of exponential variogram models fitted to the residuals of the PLS regressions using the

3 likfit function in the geoR package for the R system (http://cran.r-

4 project.org/web/packages/geoR).

\section{$5 \quad 4.4$ Residual covariance modeling}

6 The hierarchical model of equations (1)-(3) involves a spatial covariance matrix $\Sigma_{\varepsilon}\left(\phi_{\varepsilon}\right)$ for 7 the spatio-temporal residuals in equation (2). The structure of, $\Sigma_{\varepsilon}\left(\phi_{\varepsilon}\right)$, is estimated using the

8 Sampson-Guttorp deformation model for nonstationary spatial covariance fitted to an empirical

9 spatial covariance matrix computed from the spatio-temporal matrix of residuals from the site-

10 specific trend models for the AQS monitoring sites. That is, we compute residuals

$$
\hat{\varepsilon}_{i t}=\hat{\varepsilon}\left(s_{i}, t\right)=Y_{s_{i}, t}-\hat{\mu}\left(s_{i}, t\right), i=1, \ldots, N
$$

12 where

$$
\hat{\mu}\left(s_{i}, t\right)=\hat{\beta}_{0 s_{i}}+\sum_{j=1}^{m} \hat{\beta}_{j s_{i}} f_{j}(t)
$$

14 with parameter estimates $\hat{\beta}_{0 s_{i}}, \hat{\beta}_{1 s_{i}}, \ldots, \hat{\beta}_{m s_{i}}$ computed at these AQS sites as explained in section

15 4.2. We compute a covariance matrix with elements $s_{i j}=\operatorname{cov}\left(\hat{\varepsilon}_{i t}, \hat{\varepsilon}_{j t}\right)$ based on empirical

16 covariances over time between time series of residuals at locations $s_{i}$ and $s_{j}$. Because of

17 possibly substantial amounts of missing data, we use an EM algorithm to estimate this spatial

18 covariance matrix from an incomplete data matrix. The spatial deformation model expresses

19 spatial correlations as

$$
\operatorname{cor}\left(\varepsilon\left(s_{i}, t\right), \varepsilon\left(s_{j}, t\right)\right)=\gamma_{\theta}\left(\left|d\left(s_{i}\right)-d\left(s_{j}\right)\right|\right)
$$

21 where $d(s)$ is a smooth deformation of the coordinate system and $\gamma_{\theta}(h)$ is the exponential

22 spatial correlation model with parameter $\theta$. We fit this model, estimating $\theta$ and the smooth

23 deformation $d(s)$ as a pair of thin-plate splines, using the Bayesian computations explained and

24 illustrated in Damian et al $(2001,2003)$. 
We predict concentrations at subject homes using the hierarchical model described above.

3 Our methodology incorporates covariates and spatial interpolation of long-term averages and

4 seasonal trends based a on spatial correlation model, a procedure that may be regarded as a

5 generalization of "universal kriging" or "kriging with external drift" (Wackernagel 2010).

6 Specifically, given estimates of the parameters of the components of the hierarchical model as

7 explained in sections 4.1-4.4, spatio-temporal predictions at target locations $s_{0}$ are computed as

$$
\hat{Y}_{s_{0}, t}=\hat{\mu}\left(s_{0}, t\right)+\hat{\varepsilon}\left(s_{0}, t\right)
$$

where

$$
\hat{\mu}\left(s_{0}, t\right)=\hat{\beta}_{0 s_{0}}+\sum_{j=1}^{m} \hat{\beta}_{j s_{0}} f_{j}(t)
$$

11 Each of the $\hat{\beta}_{j s_{0}}$ is computed by universal kriging using the PLS component scores as spatial

12 covariates and the spatial covariance models underlying the estimates of the matrices $\Sigma_{e}\left(\phi_{j}\right)$.

13 The spatio-temporal residual field $\hat{\varepsilon}\left(s_{0}, t\right)$, being mean zero, is computed by a simple kriging

14 calculation with the spatial covariances defined by the spatial deformation model underlying the

15 spatial covariance matrix $\Sigma_{\varepsilon}\left(\phi_{\varepsilon}\right)$. Estimates are computed for each 2-week period indexed by $t$

16 using all monitoring data available at that time point from the AQS, MESA Air fixed, and MESA

17 Air home monitors.

\section{5. Application}

19 Table 1 presents descriptive statistics for $\mathrm{PM}_{2.5}$ concentrations and key spatial covariates

20 including proximities to highways, distance to commercial properties, and median population

21 densities. $\mathrm{PM}_{2.5}$ concentrations are clearly highest, on average, around Los Angeles and lowest

22 in St. Paul. Home sites in New York are closest to A1 highways while very few homes in Los

23 Angeles and Winston-Salem are near highways. Homes in Chicago are closest to commercial

24 properties. Population density is (obviously) highest in New York City and lowest in Winston-

\section{Salem.}


The detailed results that follow, illustrated in Figures 4-8, concern only the Los Angeles

2 modeling region. Figure 4 presents the results of the calculation of the SEOFs by the algorithm

3 of section 4.1. The unsmoothed EOFs include a lot of short temporal scale variation and there is

4 a considerable loss of explanatory power as a result of the smoothing. This short temporal scale

5 variation is accounted for separately in the spatio-temporal residuals, allowing us to focus the

6 modeling of the effects of spatial covariates (below) on smooth temporal trend patterns. The

7 unsmoothed first singular vector explains over $70 \%$ of the variation in the 24 log-transformed

8 AQS time series while the SEOF explains only 27.5\%. This first component reflects a long-term

9 decrease in $\mathrm{PM}_{2.5}$ levels across most of the sites over these six years and seasonal structure that is

10 quite variable from year-to-year. The second SEOF, explaining only $9.4 \%$ of the variation in the

11 original time series, turns out to describe a relatively simple seasonal structure. The nature of

12 these SEOFs varies across the four modeling regions. In North Carolina, for example, the

13 dominant SEOF is a simple seasonal pattern like that of the second SEOF here (data not shown).

14 In all cases, fitted trends based on these SEOFs, such as those illustrated in Figure 3, leave

15 residuals with very little temporal autocorrelation (results not shown here).

--- Figure 4 ---

17 The cross-validatory assessment of the number of PLS components for our land use 18 regressions based on universal kriging predictions suggests at most two predictive components 19 for all of the trend coefficients in all regions. This result is strikingly different from the result of

20 conventional PLS regression cross-validation without a spatial correlation model. As many as 10

21 PLS components are suggested for the intercept parameter $\beta_{0}$ by conventional cross-validation,

22 but substantially better cross-validatory predictions are found using only two components

23 together with a spatial correlation model.

24 Loadings (correlations) of the spatial covariates on the two PLS components for the spatial 25 regression model for the intercept (long-term mean), $\beta_{0 s}$, are illustrated in Figure 5. We see that 26 the first component is highly negatively correlated with distance to the nearest A1 highway and 27 positively correlated with the distances to the nearest A2 and A3 highways. Lengths of A1 vs 
$1 \quad \mathrm{~A} 2$ and A3 roads in buffers show the expected opposite correlation pattern. That is, this first

2 component, which is predictive of long-term mean concentrations, defines a score that contrasts

3 sites relatively near A1 highways but not necessarily near A2 and/or A3 roads with sites that are

4 near A2 and /or A3 roads but far from A1 highways. The former sites have higher $\mathrm{PM}_{2.5}$

5 concentrations on average while the latter sites have lower concentrations. The sites with

6 positive scores (near A1 highways) are largely residential as indicated by the positive loadings

7 on the residential land use covariates and contrasting negative correlations with the cropland

8 covariates. The second PLS component defines a commercial vs. residential property contrast.

--- Figure 5 ---

Scatterplots of cross-validated universal kriging predictions using two component PLS

11 regressions for each of the three trend coefficients are presented in Figure 6. The red dots are the

12 MESA Air fixed sites. We see reasonably good regressions for the long-term mean $\hat{\beta}_{0 s}$ and the

$133^{\text {rd }}$ coefficient $\hat{\beta}_{2 s}$ for the amplitude of the simple cyclic seasonal structure of Figure 4 , but little

14 ability to predict variation in the $2^{\text {nd }}$ trend coefficient $\hat{\beta}_{1 s}$ for the component carrying the long-

15 term decrease in concentrations.

--- Figure 6 ---

17 The strength of the spatial correlation structure in the deviations from the fitted trends is

18 illustrated in Figure 7. The lower left panel shows inter-site correlations vs inter-site distance for

19 the geographic configuration of sites given in the upper left panel. The horizontal axis has units

20 of 100s of kms. After a Sampson-Guttorp deformation of the geographic coordinate system,

21 illustrated in the upper right panel, we obtain the lower right scatterplot, which shows a much

22 clearer spatial correlation structure. Note that correlations do not die out to zero even over the 23 greatest spatial separation.

--- Figure 7 ---

Figure 8 presents cross-validated predictions of the 2-week observations for the four sites

26 seen in Figure 2. The dashed black lines represent the long-term means of the trend models

27 fitted to the data (black dots) while the dashed red lines are the long-term means of the cross- 
1 validation predictions (green lines varying about predicted trends drawn in red). The predictions

2 generally track the observations quite well with modest levels of over- or under-estimation of the

3 long-term means. We note that the magnitude of the error in these cross-validated predictions is

4 probably greater than the error of prediction expected at most MESA subject homes as, for

5 example, prediction of concentrations at locations near site 060372005 will benefit from the

6 monitoring data at that site, data which were excluded in its own prediction.

--- Figure 8 ---

8 Model fitting and model predictions as illustrated above for the MESA Air Southern

9 California study area were carried out similarly for the other three geographic modeling domains,

10 the Midwest region spanning Minneapolis-St Paul and Chicago, the Northeast region spanning

11 Baltimore and New York City, and North Carolina. These regions are depicted in Figures 9-11.

12 Prediction of long-term averages at the locations of the MESA Air subjects in all six study areas

13 are summarized in the boxplots of Figure 12. similar ranges of concentrations while there is relatively little variation in concentration levels

22 for the MESA Air subjects in and around Winston-Salem, NC.

23 Conventional "plug-in" estimates of the standard errors of predictions of long-term averages

24 could be computed for the final step of our prediction strategy (section 4.5). However, we

25 refrain from computing these estimates as the current model has some recognized deficiencies

26 and because such standard errors do not account for prediction uncertainty that derives from the

27 multi-step pragmatic model-fitting procedure we have employed. We report instead the 
1 accuracy of these pragmatic ambient concentration predictions in terms of descriptive statistics

2 on the cross-validated (leave-one-out) errors of prediction of long-term mean concentrations for

36 MESA Air study areas.

4 Table 2 reports first (in rows one and two) means and standard deviations for the fitted long-

5 term mean concentrations across all the AQS and MESA Air monitoring sites in each of the

6 study regions. We then report in row three the root-mean-square error of the cross-validated

7 predictions at these sites. The maps in Figures 1 and 9-11 show that these descriptive statistics

8 pertain to monitoring sites over geographic areas substantially exceeding the spatial domain of

9 the MESA Air subjects. We therefore computed these same summary statistics also on just the

10 MESA Air fixed sites, which were located within the domains of the MESA Air subjects. These

11 sites provide more relevant characterizations of the accuracy of our model predictions, albeit for

12 a relatively small number of sites in each region.

13 We see that the uncertainty of long-term estimates is similar across regions, about $3 \%$ to $5 \%$

14 of the mean. Comparison of standard deviations of long-term means to cross-validated root-

15 mean-squared errors indicates that predictions are capturing meaningful intra-urban spatial

16 variability in some areas including California, but these root-mean-square errors are nearly as

17 large as the standard deviations in some of the other study areas such as North Carolina. In

18 future work we expect improved city-specific spatio-temporal predictions and lower uncertainty

19 using new GIS computations and additional land use and traffic covariates. Furthermore,

20 uncertainty will be assessed by model-based standard errors using the likelihood modeling

21 computations of Szpiro et al. (2009) and Lindström et al. (2010) as well as cross-validation. 


\section{Discussion}

The current pragmatic $\mathrm{PM}_{2.5}$ modeling and long-term ambient concentration predictions

3 described here are based on the first phase of data collection by the MESA Air study. Revised

4 analyses will be based on monitoring data complete through 2009 and improved measures of our

5 GIS-based covariates including measures of traffic volumes not available for the current

6 analyses.

7 There are a number of important contributions of the data analyses and results presented

8 here. We have obtained insight into model selection, including the importance of accounting for

9 the spatial correlation model in using cross-validation to select the number of PLS components

10 for the mean model. We benefitted by using the MESA Air supplemental monitoring data in

11 addition to AQS data to determine estimates of model parameters $\hat{\beta}_{0 s}, \hat{\beta}_{1 s}, \hat{\beta}_{2 s}$ at both AQS and

12 MESA Air fixed sites for this regression modeling. Estimates of long-term average ambient

$13 \mathrm{PM}_{2.5}$ exposure described here are being used in preliminary health effect analyses with the

14 MESA Air cohort (Adar et al., 2009, Krishnan et al., 2009).

15 We will ultimately use the likelihood method reported in Szpiro et al. (2009) and Lindström

16 et al. (2010) because it provides a unified framework and gives standard errors (as opposed to

17 cross-validation in this paper). However, much of the model selection work will still be done

18 outside of the likelihood framework using the methods presented in this paper. This includes the

19 specification of the SEOFs and the PLS or selection of covariates for a similar regression model

20 with a universal kriging cross-validation approach. The work here, carried out in parallel to the

21 development of the likelihood method, provided the most pragmatic approach to obtaining initial

22 ambient concentration estimates for use in our epidemiology studies.

23 The current modeling and analysis leaves some problematic issues to be addressed in future

24 work. The most important is dealing appropriately with the fact that 2 -week average

25 concentrations derive from different monitoring networks (AQS and MESA Air) with different

26 monitoring instruments and temporal sampling protocols. This will require a nested 
1 specification of spatio-temporal correlation at a daily time scale. This daily time scale structure

2 is also fundamental to a "downscaling" extension of the current model predictions in order to

3 obtain estimates at a daily time scale for acute exposure estimation in epidemiologic outcomes

4 and, especially cardiovascular events that are expected to be sensitive to acute as well as chronic

5 exposure.

6 The current model includes only temporal factors (the temporal basis functions) and

7 spatially varying covariates. Recent extensions of the likelihood model fitting can incorporate

8 spatio-temporal covariates, the most important of which are spatio-temporally varying

9 characterizations of the effects of traffic on ambient exposure. Lindström et al. (2010) models

$10 \quad \mathrm{NO}_{\mathrm{x}}$ (rather than $\mathrm{PM}_{2.5}$ ) using as a covariate theoretical predictions of pollutant concentrations

11 provided by a physics-based plume dispersion model, EPA's CALINE model (Wilton et al.

12 2009).

13 The ultimate objective of the modeling described here is to provide predicted exposures for

14 estimating health effects in epidemiology studies. Up to now, we have used a "plug-in"

15 approach that does not account for the additional variability resulting from uncertainty in the

16 spatio-temporal prediction procedure. We have recently developed an efficient bootstrap-based

17 approach to incorporating this uncertainty in health effect estimation (Szpiro et al. 2009a). In

18 future work, we will apply this methodology based on fitting of the current spatio-temporal

19 model using likelihood methods in order to obtain corrected standard errors for the disease model

20 parameters of interest.

\section{Acknowledgements}

23 Although the research described in this presentation has been funded in part by the United 24 States Environmental Protection Agency through grant R831697 and assistance agreement CR2583407101 to the University of Washington, it has not been subjected to the Agency's required 26 peer and policy review and therefore does not necessarily reflect the views of the Agency and no 
official endorsement should be inferred. Additional support was provided by an award to the

2 University of Washington under the National Particle Component Toxicity (NPACT) initiative

3 of the Health Effects Institute (HEI).

\section{References}

Abdi, H. 2010. Partial least squares regression and projection on latent structure regression (PLS regression). WIREs Computational Statistics 2, 97-106.

Adar, S.D., Klein, R., Klein, B.E.K., Szpiro, A.A., Cotch, M.F., Wong, T.Y., O’Neill, M.S., Shrager, S., Graham Barr, R., Siscovick, D., Daviglus, M.L., Sampson, P.D., Kaufman, J., 2010. Air pollution and the human microvasculature in vivo assessed via retinal imaging: The Multi-Ethnic Study of Atherosclerosis and Air pollution (MESA Air), accepted for publication in PLoS Medicine.

Banerjee, S., Gelfand, A.E., Carlin P., 2004, Hierarchical Modeling and Analysis for Spatial Data. Chapman and Hall, 472 pp.

Banerjee, S., Johanson, G.A., 2006, Coregionalized single- and multiresolution spatially varying growth curve modeling with application to weed growth. Biometrics 62, 864-876.

Beckers J., Rixen, M., 2003. EOF calculations and data filling from incomplete oceanographic datasets. Journal of Atmospheric and Oceanic Technology 20, 1839-1856.

Cohen, M.A., Adar, A.D., Allen, R.W., Avol, E., Curl, C.L., Gould, T., Hardie, D., Ho, A., Kinney, P., Larson, T.V., Sampson, P.D., Sheppard, L., Stukovsky, K.D., Swan, S.S., Liu, L-J. S., Kaufman, J.D., 2009. Approach to estimating participant pollutant exposures in the Multi-Ethnic Study of Atherosclerosis and Air Pollution (MESA Air). Environmental Science \& Technology 43, 4687-4693.

Damian, D., Sampson, P.D., Guttorp, P., 2001. Bayesian estimation of semi-parametric nonstationary spatial covariance structures, Environmetrics 12, 161-178.

Damian, D., Sampson, P.D., Guttorp, P., 2003. Variance modeling for nonstationary processes with temporal replications. Journal of Geophysical Research - Atmosphere, 108 (D24). 
EPA, 2010. The U.S. Environmental Protection Agency, Technology Transfer Network (TTN)

Air Quality System (AQS), http://www.epa.gov/ttn/airs/airsaqs/ (last accessed, Sept 2010).

3 ESRI. ArcGIS (Version 9.1). Redlands, CA.

4 Fanshawe, T.R., Diggle, P.J., Rushton, S., Sanderson, R., Lurz, P.W.W., Glinianaia, S.V., Pearce, M.S., Parker, L., Charlong, M., Pless-Mulloli, T., 2008. Modelling spatio-temporal variation in exposure to particulate matter: a two-stage approach. Environmetrics 19, 549566.

Fuentes, M., Guttorp, P., Sampson, P.D., 2006. Using transforms to analyze space-time processes, in: Finkenstadt, B., Held, L., Isham, V. (Eds.), Statistical Methods for SpatioTemporal Systems, Chapman and Hall/CRC, Boca Raton, FL, USA, pp. 77-150.

Furrer, E.M., Nychka, D.W., 2007. A framework to understand the asymptotic properties of Kriging and splines. Journal of the Korean Statistical Society 36, 57-76.

Garthwaite, P. 1994. An interpretation of partial least squares. Journal of the American Statistical Association 89, 122-127.

Hoek, G., Beelen, R., de Hoogh, K., Vienneau, D., Gulliver, J., Fischer, P., Briggs, D. 2008. A review of land-use regression models to assess spatial variation of outdoor air pollution. Atmospheric Environment 42, 7561-7578.

Kent, J.T., Mardia, K.V. 1994. The link between kriging and thin-plate splines, in: Kelly, F.P. (Ed.), Probability, Statistics, and Optimization: a Tribute to Peter Whittle, Wiley, Chichester, pp. 325-339.

Krishnan, R.M., Adar, S.D., O’Neil, M.S., Barr, G.R., Polak, J.F., Herrington, D., Jorgensen, N.S., Van Hee, V.C., Kaufman, J.D., 2009. Vascular responses to short and long-term ambient air pollution: The Multi-Ethnic Study of Atherosclerosis and Air Pollution, Circulation 120, Suppl 2, S462.

Le, N.D., Zidek, J.V., 2006. Statistical Analysis of Environmental Space-Time Processes, Springer, New York, NY, USA. 
Lindström, J., Szpiro, A.A., Sheppard, L., Sampson, P.D., Oron, A., Richards, M., Larson, T., 2010. A flexible spatio-temporal model for air pollution: incorporating the output from a source dispersion model.

Moore, D.K., Jerrett, M., Mack, W.J., Kunzli, N., 2007. A land use regression model for predicting ambient fine particulate matter across Los Angeles, CA. Journal of Environmental Monitoring 9, 246-252.

Paciorek, C.J., Yanosky, J.D., Puett, R.C., Laden, F., Suh, H.H., 2009. Practical large-scale spatio-temporal modeling of particulate matter concentrations. Annals of Applied Statistics 3, 370-397.

R Development Core Team, 2009. R: A Language and Environment for Statistical Computing. R Foundation for Statistical Computing, Vienna, Austria (http://www.Rproject.org).

Rao, S.T. Zurbenko, I.G., 1994. Detecting and tracking changes in ozone air quality, Journal of the Air \& Waste Management Association 44, 1089-1092.

Ross, Z., Jerrett, M., Ito, K., Tempalski, B., Thurston, G.D., 2007. A land use regression for predicting fine particulate matter concentrations in the New York City region. Atmospheric Environment 41, 2255-2269.

Sahu, S.K., Gelfand, A.E., Holland, D.M., 2006. Spatio-temporal modeling of fine particulate matter. Journal of Agricultural, Biological, and Environmental Statistics 11, 61-86.

Su, J.G., Jerrett, M., Beckerman, B., Wilhelm, M., Ghosh, J.K., Ritz, B., 2009. Predicting trafficrelated air pollution in Los Angeles using a distance decay regression selection strategy. Environmental Research 109, 657-670.

Smith, R.L., Kolenikov, S., Cox, L.H., 2003. Spatio-temporal modeling of $\mathrm{PM}_{2.5}$ data with missing values. Journal of Geophysical Research 108(D24), 9004.

Szpiro, A.A., Sampson, P.D., Sheppard, L., Lumley, T., Adar, S.D., Kaufman, J.D., 2009. Predicting intraurban variation in air pollution concentrations with complex spatiotemporal interactions. Environmetrics, in press. 
1 Szpiro, A.A., Sheppard, L., Lumley, T., 2009a. Efficient measurement error correction with spatially misaligned data. UW Biostatistics Working Paper Series, Working Paper 350. http://www.bepress.com/uwbiostat/paper350

4 Wackernagel, H., 2010. Multivariate Geostatistics: An Introduction with Applications, $3^{\text {rd }}$ ed. Springer-Verlag, Berlin, Germany.

6 Wilton, D., Szpiro, A., Gould, T., Larson, T., 2010. Improving spatial concentration estimates for nitrogen oxides using a hybrid meteorological dispersion/land use regression model in

8
Los Angeles, CA and Seattle,WA. Science of the Total Environment, 408 1120-1130.

Wise, E.K., Comrie, A.C., 2005. Extending the Kolmogorov-Zurbenko filter: Application to ozone, particulate matter, and meteorological trends. Journal of the Air \& Waste Management Association 55, 1208-1216.

Taylor, C.J., Pedregal, D.J., Young, P.C., Tych, W., 2006. Environmental time series analysis 3 and forecasting with the Captain toolbox. Environmental Modelling \& Software 22, 797 814.

Tibshirani, R. (1996). Regression shrinkage and selection via the lasso. Journal of the Royal Statistical Society, Series B 58, 267-288.

7 Yanosky, J.D., Paciorek, C.J., Suh, H.H., 2009. Predicting chronic fine and coarse particulate exposures using spatio-temporal models for the Northeastern and Midwestern United States. Environmental Health Perspectives 117, 522-529. 
1 Table 1. Site descriptive statistics for selected spatial covariates and average $\mathrm{PM}_{2.5}$

2 concentration.

3

\begin{tabular}{|c|c|c|c|c|c|c|c|c|c|c|}
\hline \multirow{2}{*}{$\begin{array}{l}\text { City - sites } \\
\text { Los Angeles }\end{array}$} & \multirow[t]{2}{*}{ \#sites } & \multirow[t]{2}{*}{$\begin{array}{l}\text { Min \#obs } \\
\text { /site }\end{array}$} & \multirow[t]{2}{*}{$\begin{array}{l}\text { Max \#obs } \\
\text { /site }\end{array}$} & $\begin{array}{l}\text { \#Sites }<150 \mathrm{~m} \\
\text { to A1 }(\%)\end{array}$ & $\begin{array}{l}\text { \#Sites }<150 \mathrm{~m} \\
\text { to } \mathrm{A} 3(\%)\end{array}$ & \multirow[t]{2}{*}{$\begin{array}{l}\text { Med dist to } \\
\mathrm{A} 1, \mathrm{~A} 2, \mathrm{~A} 3\end{array}$} & \multirow[t]{2}{*}{$\begin{array}{l}\text { Med dist to } \\
\text { Commerce }\end{array}$} & \multirow[t]{2}{*}{$\begin{array}{l}\text { Med Pop } \\
\text { dens }\end{array}$} & \multicolumn{2}{|c|}{$\begin{array}{l}\text { Ave PM2.5 } \\
\text { (sd) }\end{array}$} \\
\hline & & & & & & & & & & \\
\hline $\mathrm{AQS}$ & 24 & 177 & 363 & $(0)$ & $(0)$ & 671 & 28 & 1621 & 17 & (5) \\
\hline Fixed & 7 & 19 & 28 & 3 (43) & (0) & 851 & 417 & 5103 & 17 & (3) \\
\hline Home & 45 & 1 & 2 & (4) & (2) & 1049 & 274 & 3718 & 16 & (3) \\
\hline \multicolumn{11}{|l|}{ Chicago } \\
\hline $\mathrm{AQS}$ & 44 & 133 & 364 & (2) & $5 \quad(11)$ & 464 & 100 & 867 & 14 & (2) \\
\hline Fixed & 7 & 7 & 34 & 2 (29) & (0) & 338 & 0 & 1581 & 13 & (2) \\
\hline \multirow{2}{*}{\multicolumn{11}{|c|}{ St. Paul }} \\
\hline & & & & & & & & & & \\
\hline $\mathrm{AQS}$ & 41 & 88 & 365 & (2) & (5) & 902 & 188 & 1256 & 10 & (2) \\
\hline Fixed & 3 & 27 & 29 & 1 (33) & (0) & 764 & 130 & 2351 & 9 & (1) \\
\hline Home & 37 & 1 & 2 & 2 (5) & $4 \quad$ (11) & 461 & 697 & 2906 & 9 & (3) \\
\hline \multicolumn{11}{|l|}{ New York } \\
\hline $\mathrm{AQS}$ & 45 & 111 & 356 & $6 \quad$ (13) & (2) & 446 & 117 & 3207 & 13 & (1) \\
\hline Fixed & 3 & 31 & 32 & 1 (33) & $1 \quad$ (33) & 99 & 133 & 4403 & 13 & (3) \\
\hline Home & 78 & 1 & 2 & 9 (12) & (0) & 402 & 249 & 43819 & 14 & (4) \\
\hline \multicolumn{11}{|l|}{ Baltimore } \\
\hline $\mathrm{AQS}$ & 39 & 205 & 365 & (3) & $6 \quad(15)$ & 360 & 102 & 703 & 14 & (1) \\
\hline Fixed & 5 & 14 & 33 & 1 (20) & $(0)$ & 210 & 156 & 844 & 14 & (1) \\
\hline Home & 39 & 1 & 1 & 2 (5) & (5) & 418 & 372 & 1809 & 15 & (4) \\
\hline \multicolumn{11}{|l|}{ Winston-Salem } \\
\hline $\mathrm{AQS}$ & 29 & 116 & 365 & (0) & $3 \quad(10)$ & 547 & 366 & 461 & 14 & (1) \\
\hline Fixed & 4 & 18 & 35 & $1 \quad(25)$ & (0) & 208 & 478 & 481 & 14 & (1) \\
\hline Home & 52 & 1 & 2 & $(0)$ & (0) & 698 & 988 & 516 & 15 & (4) \\
\hline
\end{tabular}

5 Notes: "Fixed" and "Home" refer to MESA Air supplemental fixed and home outdoor

6 monitoring sites. The simple average and standard deviation of reported PM2.5 concentrations

7 reported here for MESA Air sites are influenced by seasonality and unbalanced temporal

8 sampling (see Fig 2) that is not accounted for and the values for the MESA Air Home sites, in

9 particular, are based on very small numbers of observations. "Med dist to A1,A2,A3" is the

10 median over sites of the minimum distance to a major road of class A1, A2, or A3. "Med dist to

11 Commerce" is the median over sites of the distance to the nearest commercial land use property.

12 "Med Pop dens" is the median over sites of the block group population density. 
1 Table 2. Descriptive statistics on the cross-validated (leave-one-out) errors of prediction of

2 long-term mean concentrations for 4 major modeling regions

3

\begin{tabular}{|c|c|c|c|c|c|c|c|}
\hline \multirow{2}{*}{$\begin{array}{l}\text { Data } \\
\text { Scale }\end{array}$} & \multirow[t]{2}{*}{ Site } & \multicolumn{3}{|c|}{ All Sites } & \multicolumn{3}{|c|}{ MESA Air Fixed Sites } \\
\hline & & Mean & SD & RMSE & Mean & SD & RMSE \\
\hline \multirow[t]{6}{*}{$\log$} & $\mathrm{CA}$ & 2.85 & 0.27 & 0.15 & 3.02 & 0.15 & 0.04 \\
\hline & $\mathrm{IL}$ & 2.66 & 0.13 & 0.09 & 2.67 & 0.12 & 0.04 \\
\hline & MN & 2.30 & 0.14 & 0.10 & 2.36 & 0.07 & 0.03 \\
\hline & MD & 2.70 & 0.08 & 0.07 & 2.76 & 0.06 & 0.03 \\
\hline & NY & 2.61 & 0.11 & 0.09 & 2.68 & 0.18 & 0.03 \\
\hline & $\mathrm{NC}$ & 2.66 & 0.06 & 0.07 & 2.69 & 0.03 & 0.03 \\
\hline \multirow[t]{6}{*}{ Original } & $\mathrm{CA}$ & 17.84 & 4.47 & 2.42 & 20.64 & 3.04 & 0.94 \\
\hline & $\mathrm{IL}$ & 14.45 & 1.89 & 1.31 & 14.59 & 1.93 & 0.68 \\
\hline & MN & 10.07 & 1.32 & 0.88 & 10.61 & 0.72 & 0.34 \\
\hline & MD & 14.90 & 1.23 & 1.05 & 15.77 & 0.90 & 0.42 \\
\hline & NY & 13.66 & 1.58 & 1.21 & 14.70 & 2.52 & 0.44 \\
\hline & $\mathrm{NC}$ & 14.34 & 0.92 & 0.95 & 14.72 & 0.39 & 0.40 \\
\hline
\end{tabular}

4 
Figure 1. Monitoring sites and subject home locations in the Los Angeles region.

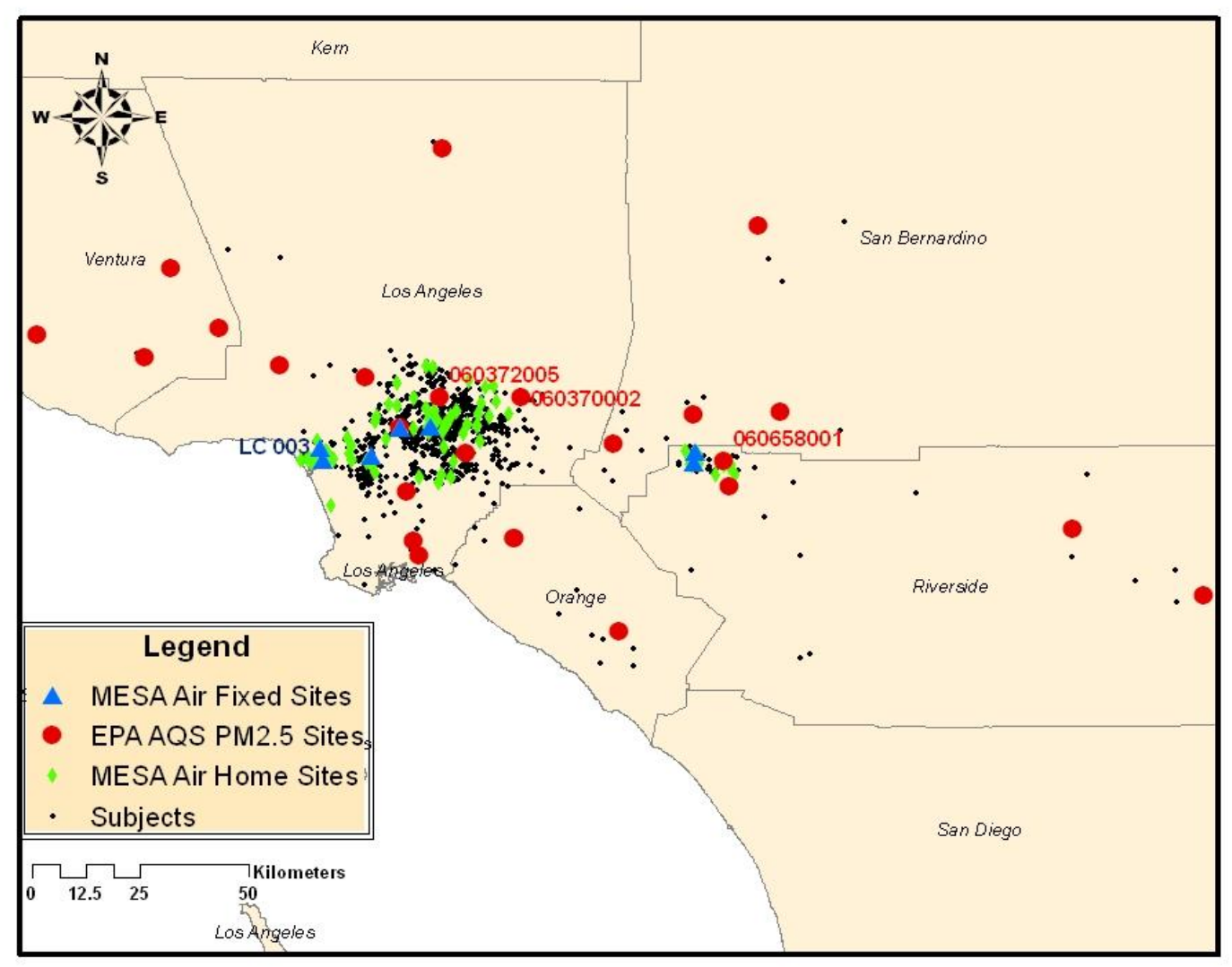


$1 \quad$ Figure 2. Schematic of the temporal sampling pattern for AQS monitors and the MESA Air 2 fixed and home sites. Each point in this figure represents a 2-week average measurement.

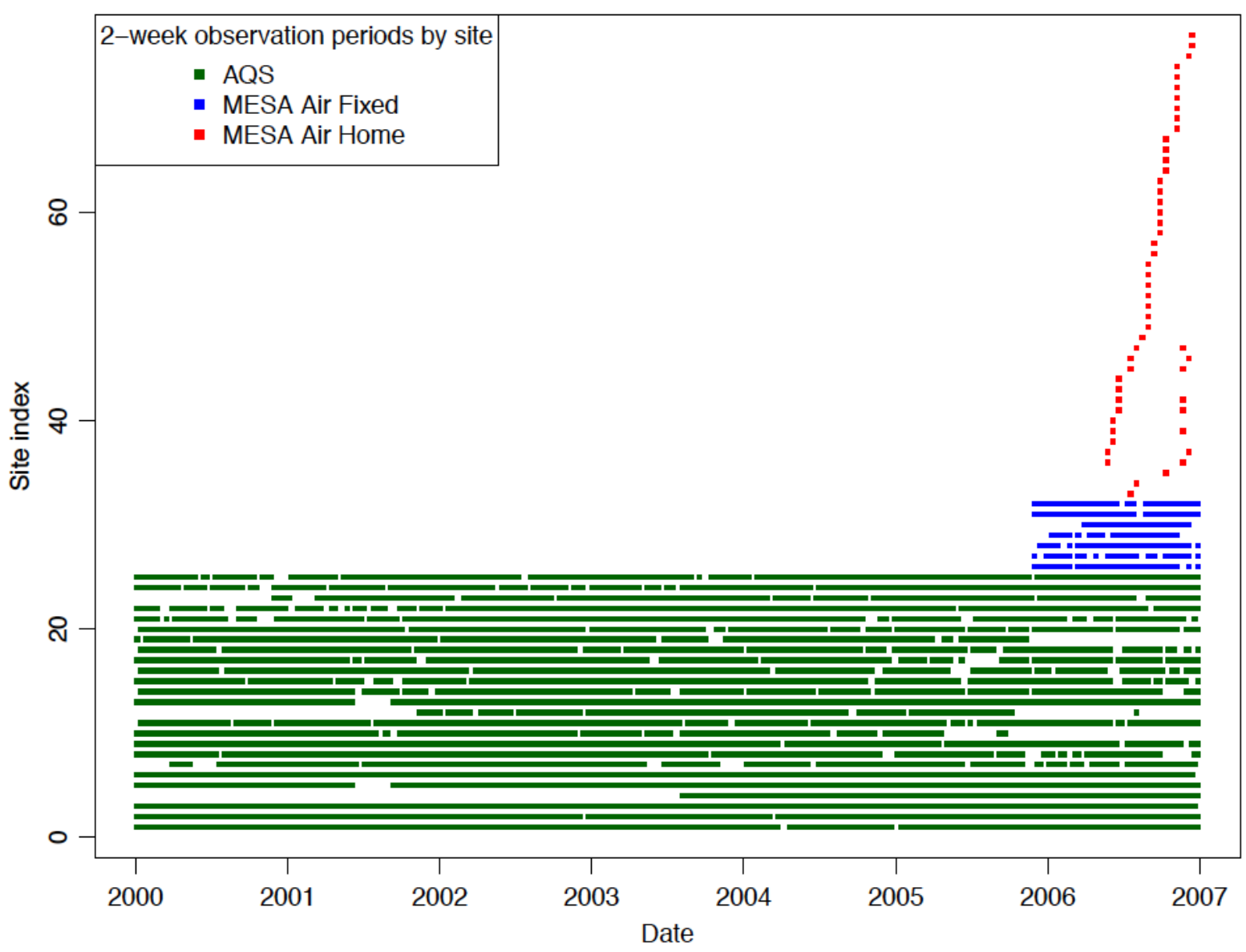


1 Figure 3. Example log-transformed two-week average $\mathrm{PM}_{2.5}$ data at AQS and MESA Air 2 fixed sites in the Los Angeles region. The black points are measurements and the lines 3 represent estimated temporal trends based on the SEOF model (see Section 4.2). The $4 \quad$ locations of each of these sites are shown on the map in Figure 1.
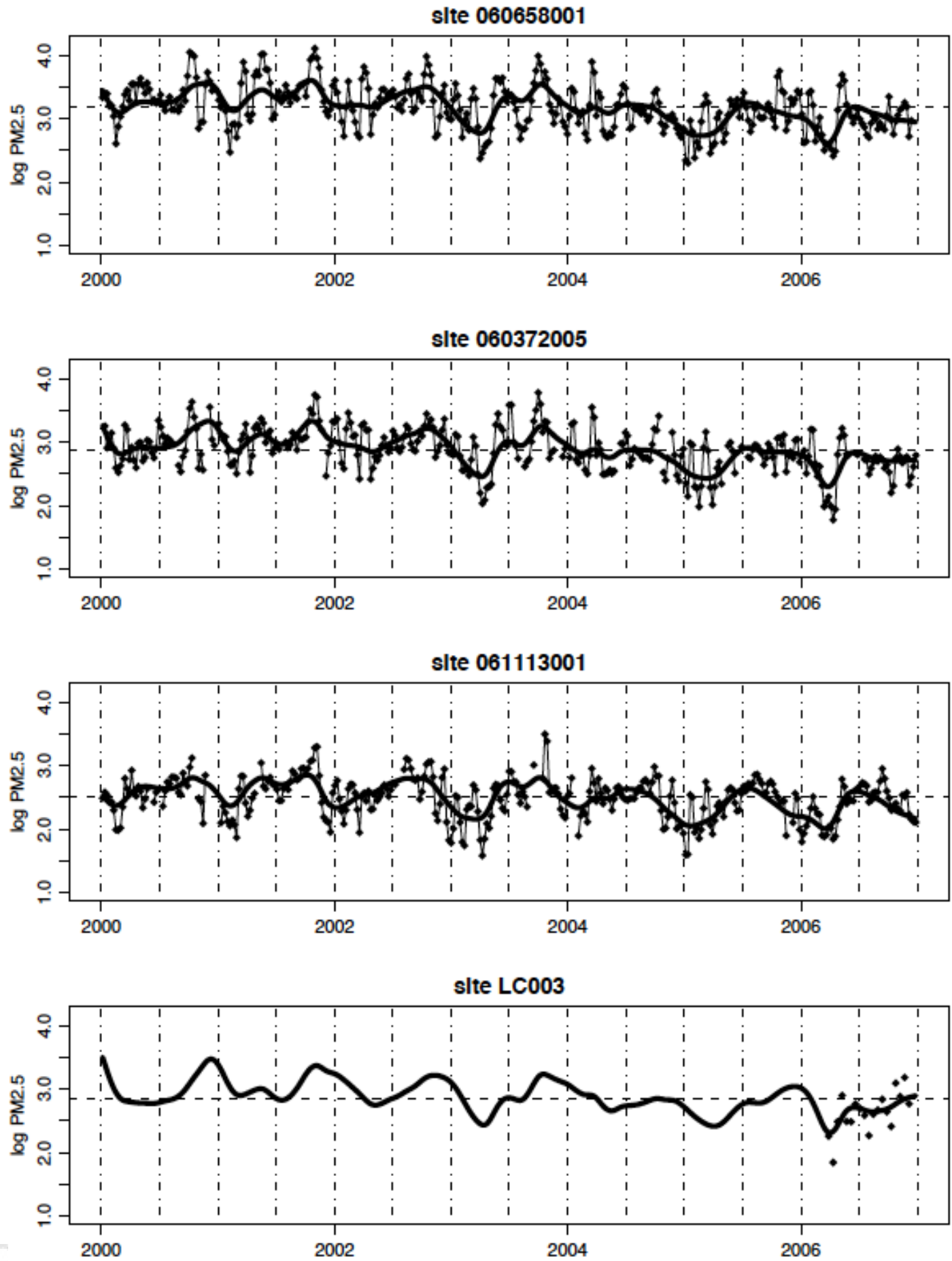
Figure 4. Smoothed empirical orthogonal basis functions for log-transformed two-week average concentrations of $\mathrm{PM}_{2.5}$ in the Los Angeles area. The first smoothed component explains $27.5 \%$ of the variation in the matrix of log-transformed 2-week average $\mathrm{PM}_{2.5}$ concentrations while the second component explains only $9.4 \%$ of the variation.

Trend component \# 1

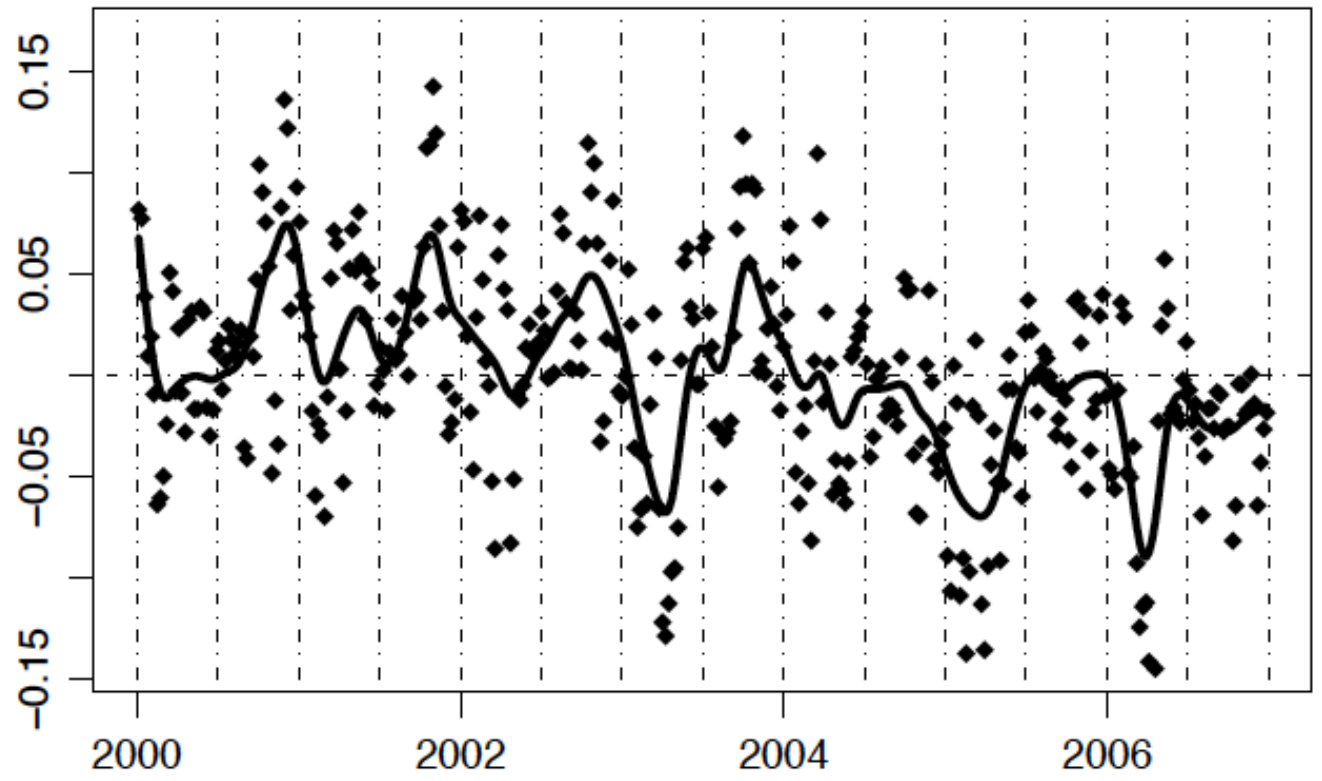

Trend component \# 2

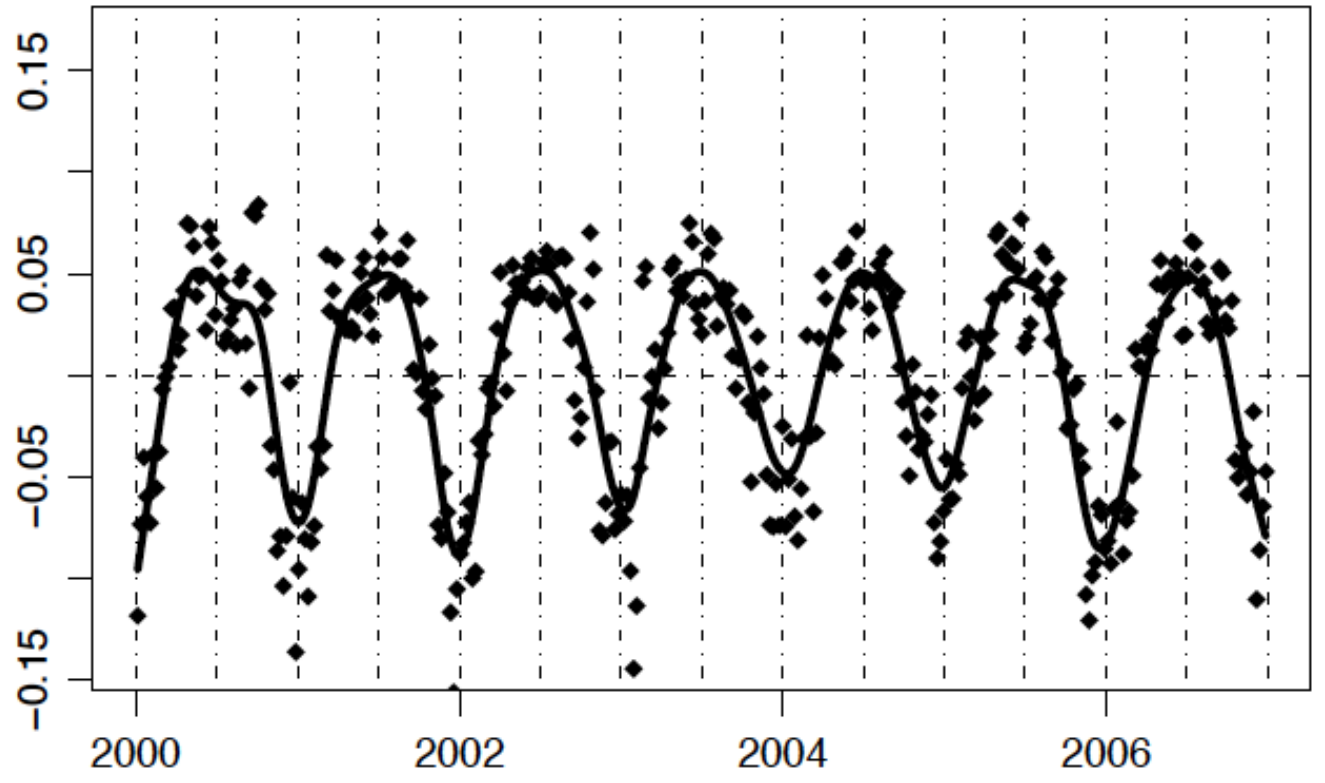


Figure 5. Loadings (correlations) of the spatial covariates on the two Partial Least Squares (PLS) components for the spatial regression model for the intercept (long-term mean), $\beta_{0 s}$, for the Los Angeles study region. The a1, a2, and a3 variables refer to major road census feature class codes; they include the angle to the nearest such roadway, the lengths of roadway segments in circular buffers of varying radii, and the distances in meters to the nearest roadway. The variables beginning COMM, CROP, INDUSTRI, and RESI refer to fractions of the property in circular buffers designated as Commercial, Cropland, Industrial, and Residential using the ArcGIS software system (ESRI).

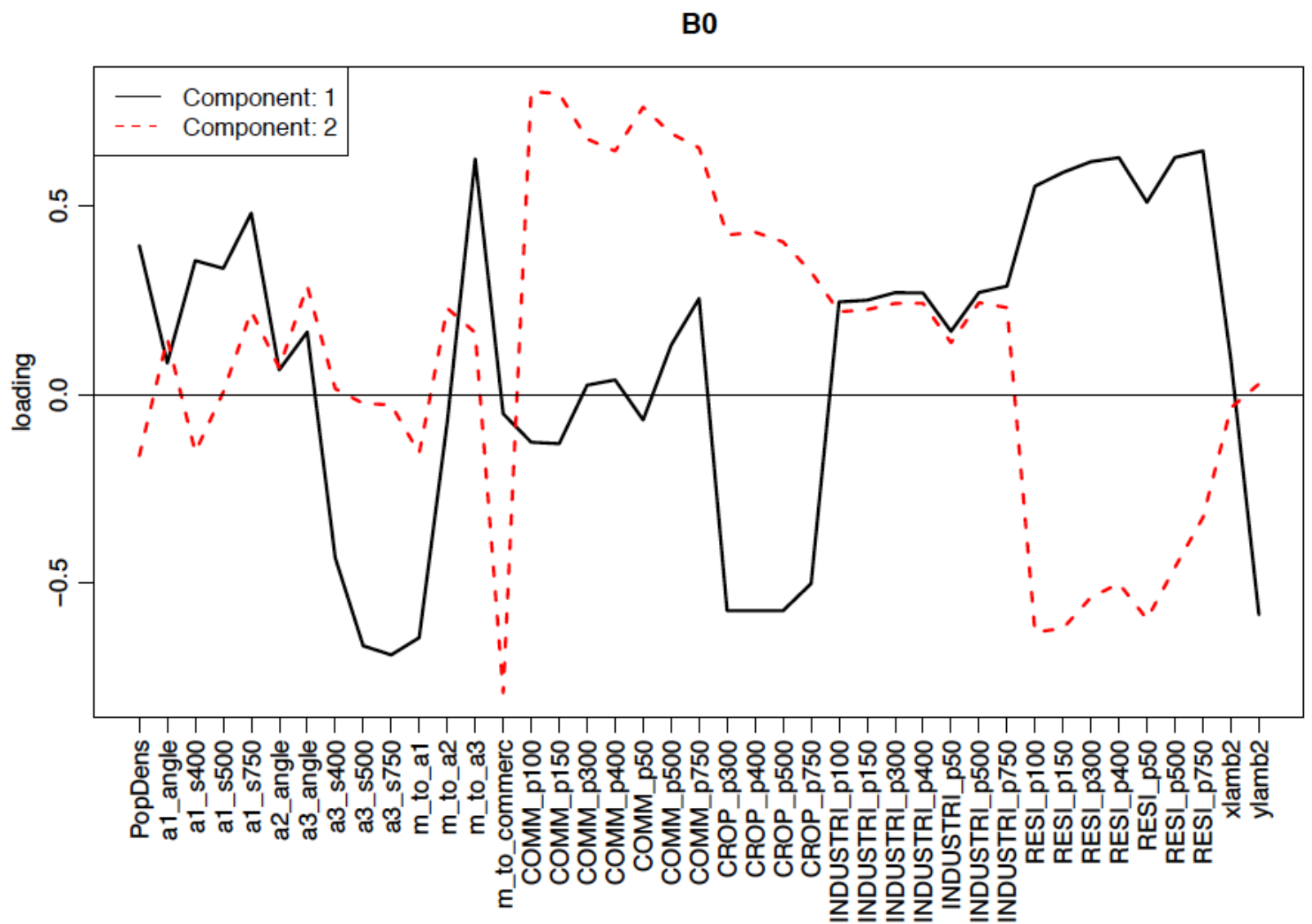


$1 \quad$ Figure 6. Cross-validated predictions of the $\beta_{0 s}, \beta_{1 s}$, and $\beta_{2 s}$ spatial fields of coefficients 2 for the long-term average and two SEOF temporal trends in the Los Angeles region. The 3 black dots represent the AQS locations while the red dots are the MESA Air fixed sites. The 4 predictions are based on PLS regression models with 2, 1, and 1 components, respectively. 5
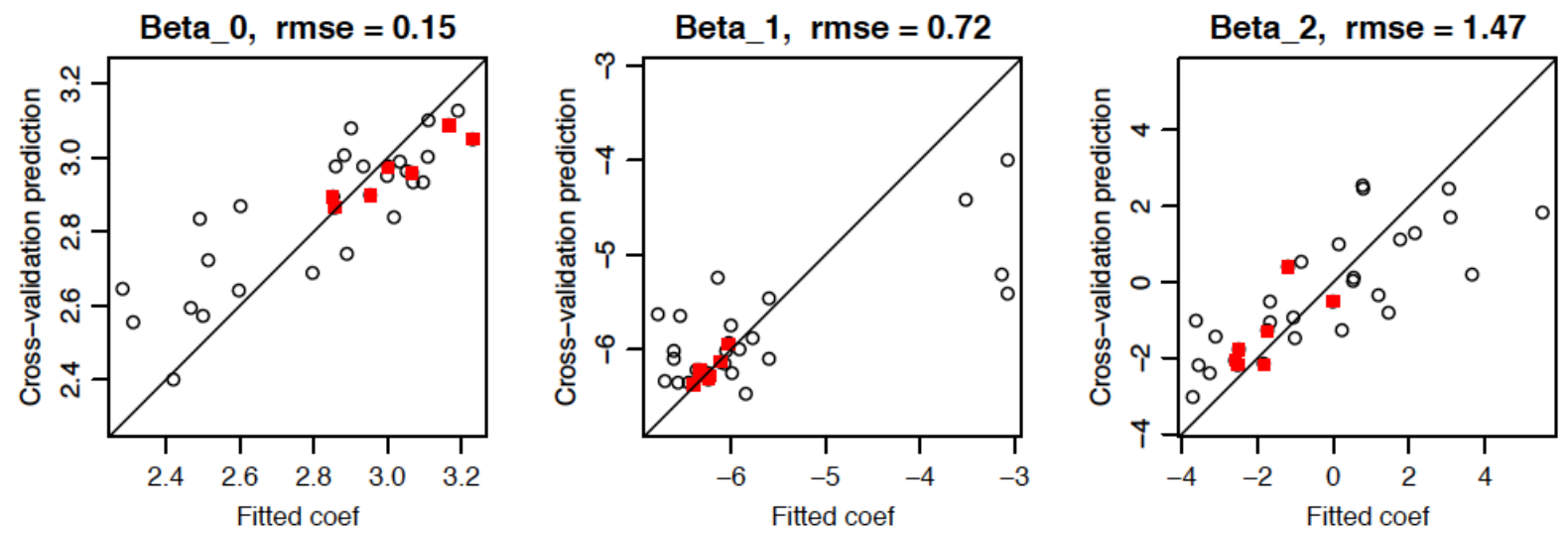
$1 \quad$ Figure 7. Spatial structure of the spatio-temporal residuals before (left) and after (right) 2 transformation using the Sampson-Guttorp method to account for nonstationarity.
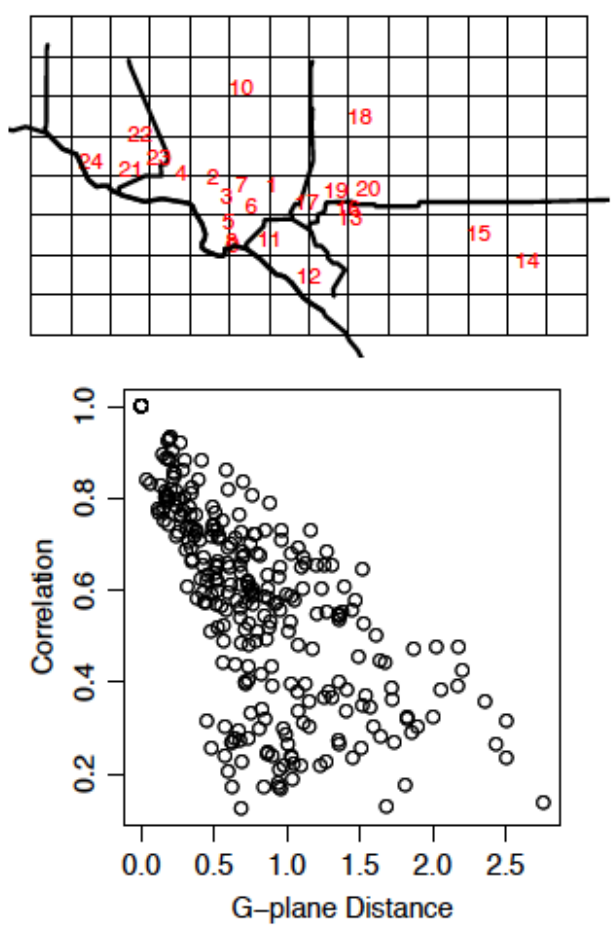
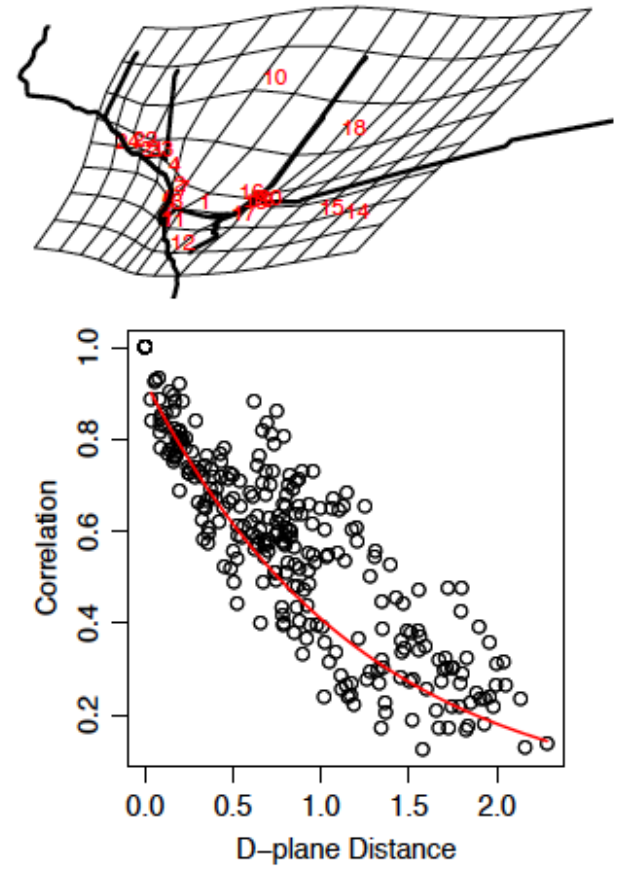
1 Figure 8. Example cross-validated predictions of log-transformed two-week concentrations at AQS and MESA Air fixed sites in the Los Angeles region. The black dots are the measured data, the red curves show predicted trends based on the SEOF part of the spatiotemporal model, and the green lines show two-week average predictions that incorporate the spatio-temporal residuals.
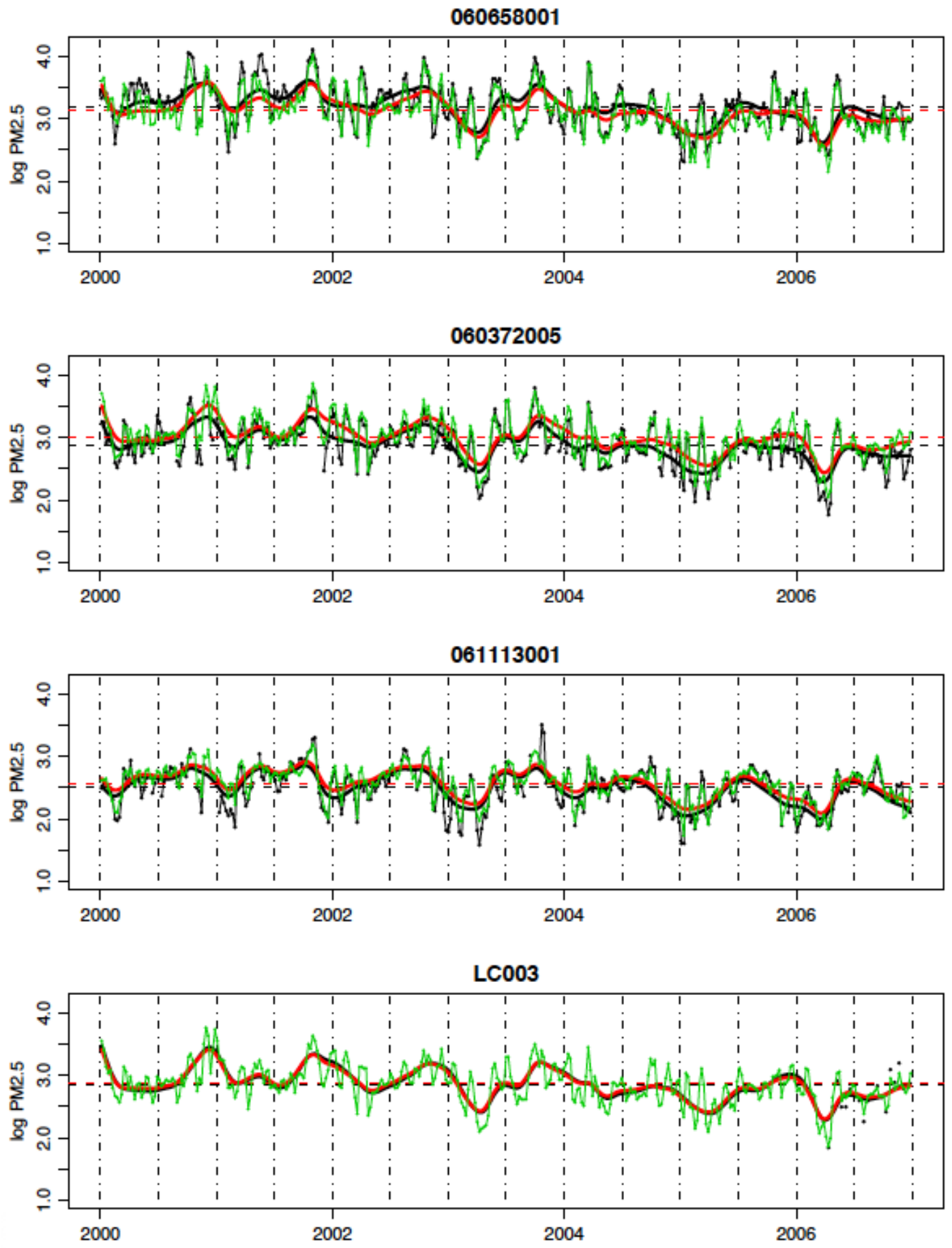
1 Figure 9. Monitoring sites and subject home locations in the midwest region spanning the 2 Minneapolis-St Paul and Chicago MESA Air study areas.

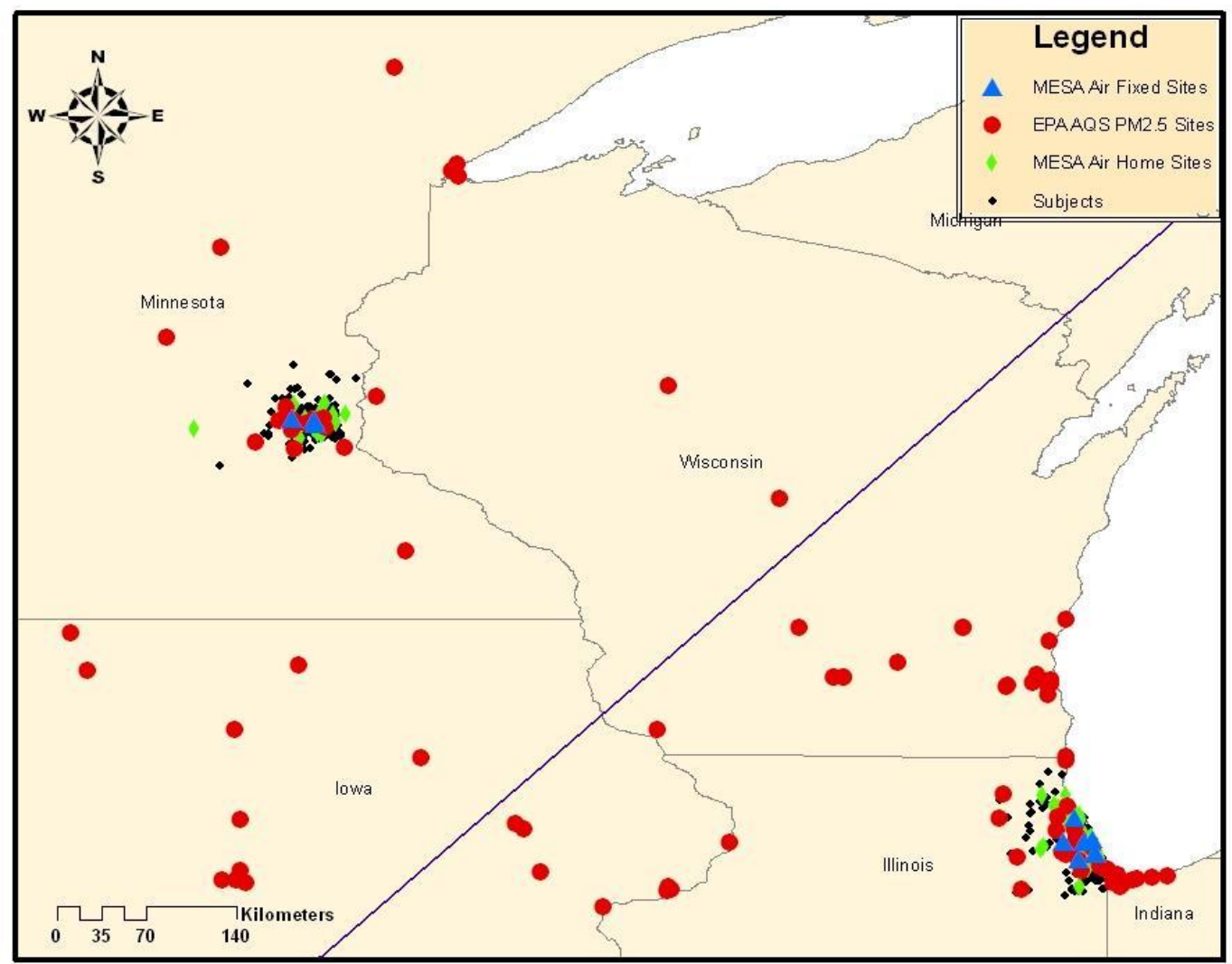


1 Figure 10. Monitoring sites and subject home locations in the northeast region spanning the New 2 York and MESA Air study areas.

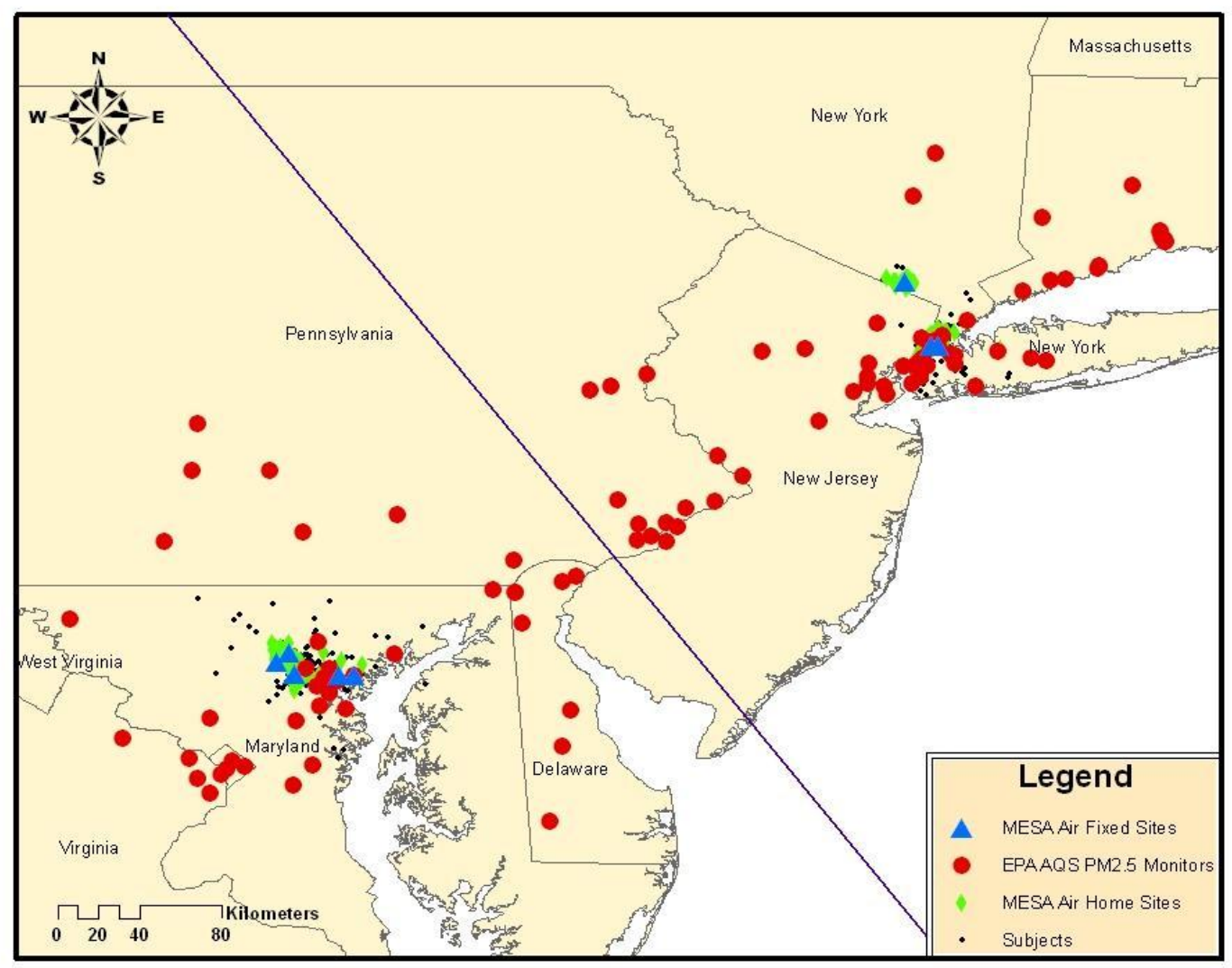

4 
Figure 11. Monitoring sites and subject home locations in the North Carolina region.

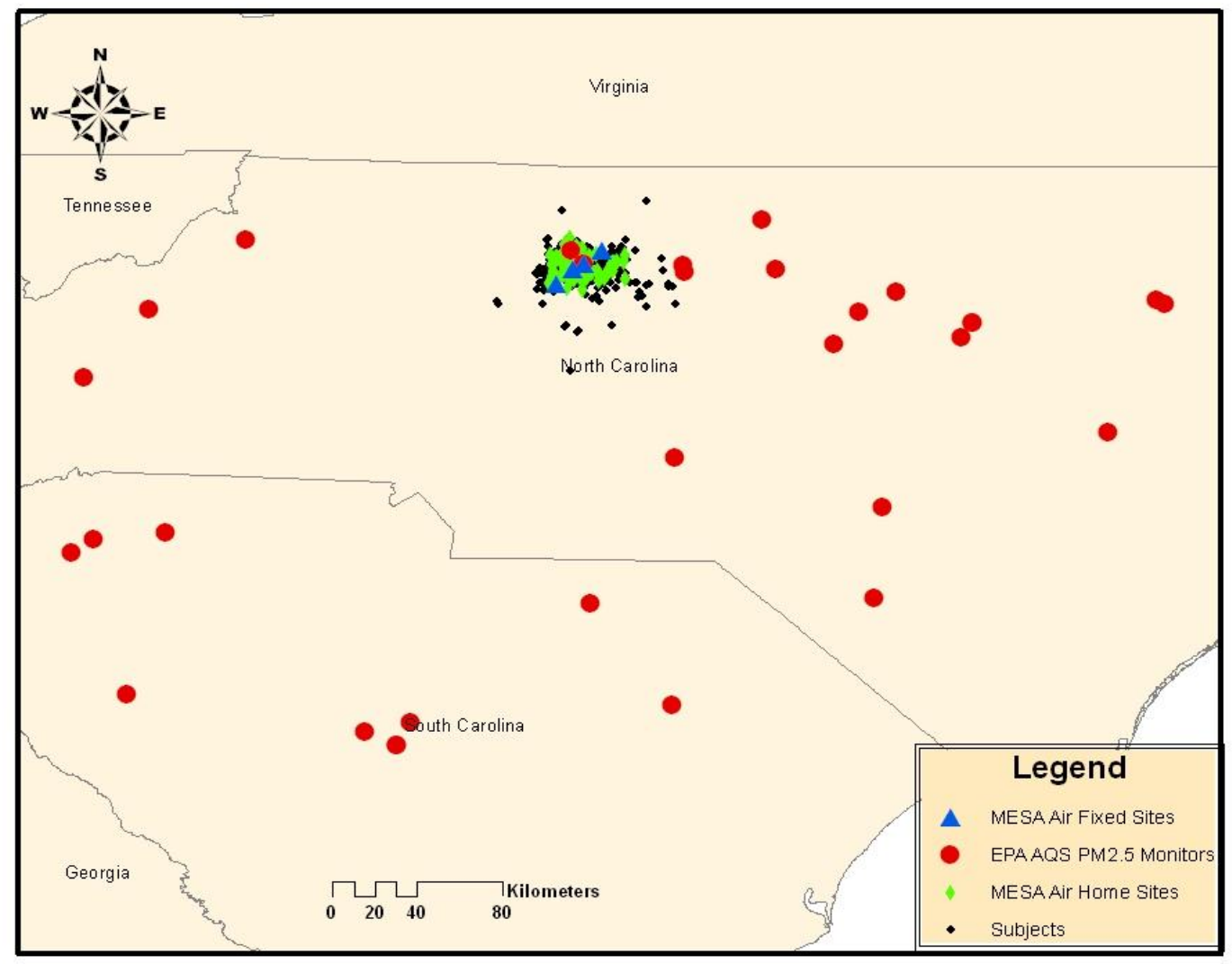


1 Figure 12. Predicted long-term average concentrations of $\mathrm{PM}_{2.5}(\mathrm{ppb})$ at all subject home 2 locations in each of the six MESA Air study areas.

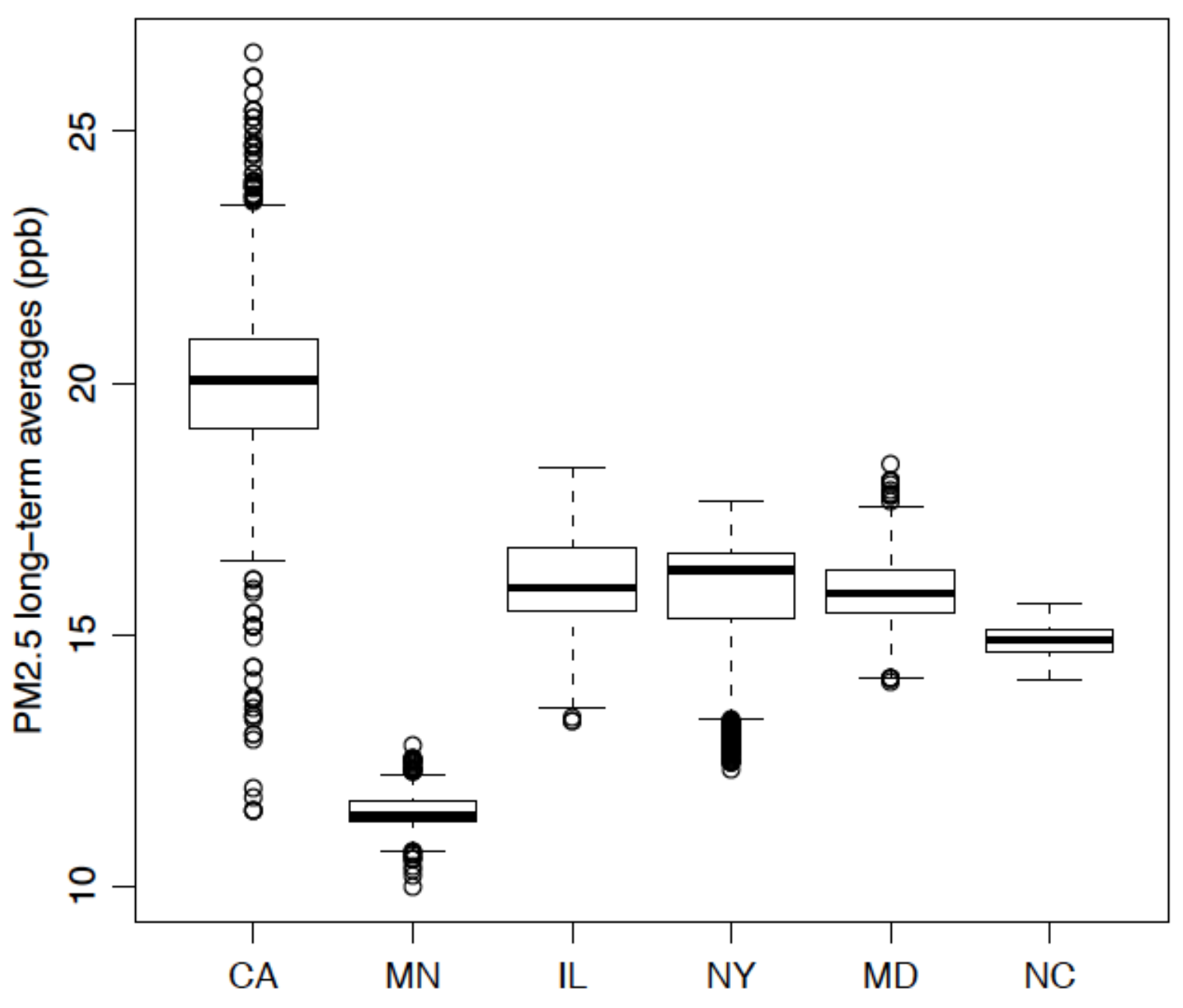

